\title{
A DFT-based theoretical model for the calculation of spectral profiles of lanthanide $\mathbf{M}_{4,5}$-edge $x$-ray absorption
}

\author{
Harry Ramanantoanina $a^{a)}$ \\ Paul Scherrer Institute, CH-5232 Villigen, Switzerland
}

(Received 5 June 2018; accepted 11 July 2018; published online 3 August 2018)

\begin{abstract}
This presentation reports the theoretical study of $3 d$ core-electron excitation in lanthanide compounds in terms of electronic structure effects and optical properties. The calculations are done at the DensityFunctional Theory (DFT) level complemented with an effective Hamiltonian based on ligand-field theory. The strategy consists of obtaining from DFT a totally symmetric density, where an active subspace is set up that forms the basis of the fivefold $3 d$ and sevenfold $4 f$ atomic orbitals of the lanthanide ion. This active subspace is defined with the fractional occupation of electrons, which represents openshell species with the composite configuration $3 d^{9} 4 f^{n+1}$. Based on the ligand-field analysis of the DFT results, the multiplet energies and ligand-field effects associated with the configuration $3 d^{9} 4 f^{n+1}$ are evaluated; and the X-ray absorption spectra are simulated in terms of the intra-atomic $4 f^{n} \rightarrow 3 d^{9} 4 f^{n+1}$ electron transitions within the electric-dipole approximation. Examples for application are proposed taking into consideration the isolated trivalent lanthanides ions and compounds $\mathrm{Cs}_{2} \mathrm{NaPrX}_{6}$, with $\mathrm{X}=\mathrm{F}, \mathrm{Cl}$, and $\mathrm{Br}$. The results are compared with available experimental data, where a good agreement is qualitatively achieved. Also, the screening of the inter-electron repulsion and spin-orbit coupling interaction is numerically obtained that allows one to establish a fully non-empirical treatment of the $3 d$ core-electron excitation, which can be valuable in the characterization and modeling of the spectral profiles of lanthanide $\mathrm{M}_{4,5}$-edge $\mathrm{X}$-ray absorption spectroscopy. The enclosed theoretical model, which is being implemented in the Amsterdam Density Functional (ADF) suite of programs, is computationally economic and can be applied to any lanthanide system without limitations in terms of the size of the matrix elements of the effective Hamiltonian or the coordination symmetry of the lanthanide center. Published by AIP Publishing. https://doi.org/10.1063/1.5043052
\end{abstract}

\section{INTRODUCTION}

Much of the most groundbreaking and technological materials reported from the last two decades contain lanthanide elements. ${ }^{1}$ For instance, the applications of lanthanides in today's technology driven life are as diverse as they are ubiquitous: neutron absorber in the nuclear reactor, phosphors in light-emitting diodes, contrast agents in magnetic resonance and imaging, catalyst in organic synthesis, magnets, etc. Lanthanides are also implicated in regulating the magnetic and optical properties of materials, which stems from unique electronic structure effects arising from highly correlated electrons within the valence orbitals.

A great deal of research activities is naturally focused on the study of lanthanides. While experimental initiative concentrates on materials' characterization and analysis by means of spectroscopic tools, there is an increasing interest to perform X-ray Absorption Spectroscopy (XAS) measurements triggered by the increased availability of high intensity synchrotron radiation as the X-ray source. ${ }^{2,3}$ The practical advantage of XAS lies in the local structure probe, giving rise to practical information at the electronic and

a)E-mail: harry.ra@hotmail.com atomistic levels. ${ }^{4}$ However even with such an advantage, the interpretation of X-ray absorption spectra is often fraught with many difficulties: i.e., the absorption process is associated with an intricate mechanism involving the creation of core-hole ${ }^{4}$ and multiple open-shell species are always encountered as intermediate or final electronic states. ${ }^{4,5}$ Therefore expertise from theoretical spectroscopy and modeling is regularly demanded at least eventually to rationalize experimental findings and to characterize complex spectral profiles.

Although in Density-Functional Theory (DFT), the timedependent formalism is in general the method of choice that deals with electronic excited states and optical properties, ${ }^{6,7}$ the explicit treatment of open-shell species remains challenging, namely, when multiplet effects and spin-orbit coupling are imposed. The lanthanide $\mathrm{M}_{4,5}$-edge XAS process yields the final electronic state with two open shell configurations, which results from the $3 d$ core-electron excitation, i.e., $3 d^{9} 4 f^{n+1}$. Decades ago, a systematic computation of the multiplet energies has been reported, ${ }^{8}$ using the effective Hamiltonian by taking only into account the interactions due to electrostatic inter-electron correlation and spin-orbit coupling. It is shown that parameters, which directly input the effective Hamiltonian, can be obtained from Hartree-Fock calculations with relativistic corrections. ${ }^{8}$ But these parameters are nonetheless adjustable since the Slater-Condon integrals are scaled down 
to $80 \%$ of their theoretical values for better reproduction of the experimental data, and the spin-orbit coupling constants are adjusted with scaling factors depending to the lanthanide ion. ${ }^{8}$ Nowadays as the research in this topic continues, the explicit control over many adjustable parameters is not trivial. This opens new developments in computational modeling, questioning the relevance of the theoretical model, whether it is ever connected with some empiricism or not.

In the present work, aiming to revisit the ligand-field concept in the frame of the modern DFT tool, a theoretical model for the study of the electronic structure and optical properties of lanthanide-based materials under X-ray radiation is proposed. The methodological concept combines the first principles DFT calculation with an effective Hamiltonian based on the principles of ligand-field theory. ${ }^{9,10}$ It includes the calculation of the multiplet energies and ligandfield splitting of the two open-shell configurations $3 d^{9} 4 f^{n+1}$ of lanthanide ions, with $n=0,1,2, \ldots$, and 13 , as well as the simulation of the excitation energies and oscillator strengths of the intra-atomic $4 f^{n} \rightarrow 3 d^{9} 4 f^{n+1}$ electron transitions within the electric-dipole approximation. Empirical corrections and scaling factors are not taken into consideration, presenting an original and non-standard DFT-based approach of the X-ray spectroscopies of lanthanide materials with good agreement with experiments. In turn, the theoretical model is aimed at complementing existing numerical tools for the modeling of core-electron excitation, important in the characterization of materials with technological potential. The present theoretical model is being implemented in the Amsterdam Density Functional (ADF) program package, ${ }^{11-13}$ as part of the recently available Ligand-Field DensityFunctional Theory (LFDFT) tool, ${ }^{14-20}$ making the resources available for broader users with experimental and theoretical interest.

\section{THEORETICAL MODEL}

\section{A. DFT calculation}

The DFT calculation procedure uses the concept of the average of configuration (AOC) method, ${ }^{21}$ which allows one to obtain a statistically averaged electron density in Slater's sense that is well suited to resolve ligand-field states. ${ }^{14-20}$ The AOC method forces fractional occupation of electrons within Kohn-Sham orbitals that are selectively chosen as the active subspace. In the present work, this active subspace forms the basis of the fivefold $3 d$ and sevenfold $4 f$ atomic orbitals of the lanthanide ion. The DFT run is a restricted Self-Consistent Field (SCF) calculation, without symmetry constraint, where the Kohn-Sham orbitals assimilated with dominant $3 d$ and $4 f$ characters of the lanthanide ion are populated with $9 / 5$ and $(n+1) / 7$ electrons, respectively, that $a$ priori represents the composite configuration system, $3 d^{9} 4 f^{n+1}$, under consideration. The results of such a DFT calculation yield electron density that belongs to the totally symmetric representation under which the effective ligand-field Hamiltonian is invariant. Figure 1 shows the DFT results of the electronic structure of the isolated $\mathrm{Pr}^{3+}$ ion within the configuration $3 d^{9} 4 f^{3}$. The calculated electronic structure is compared to that obtained

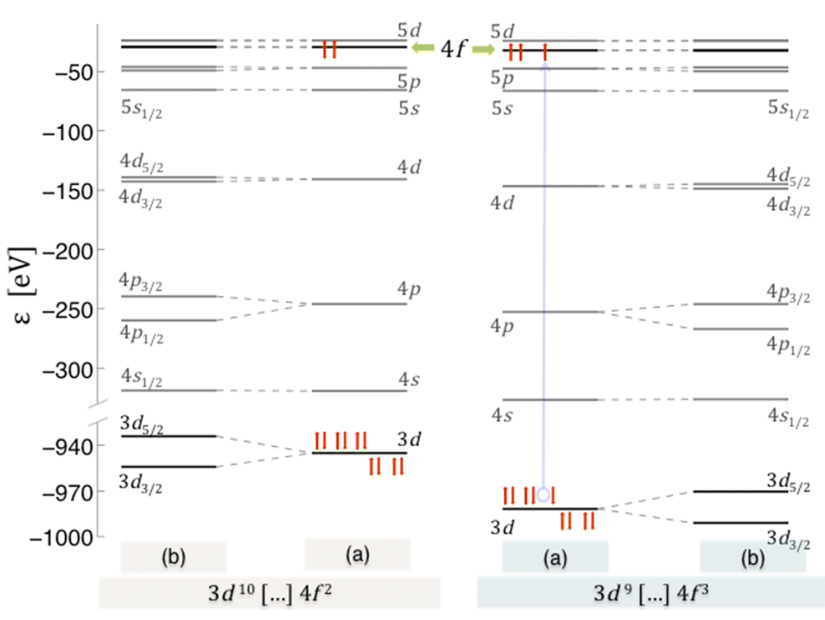

FIG. 1. Kohn-Sham orbital energy diagrams of the $\mathrm{Pr}^{3+}$ ion obtained by DFT using the GGA functional and relativistic ZORA corrections at both scalar (a) and spin-orbit (b) levels of theory. The left-hand and right-hand columns show the energy levels obtained for configurations $4 f^{2}$ and $3 d^{9} 4 f^{3}$, respectively.

within $4 f^{2}$ that represents the ground state electron configuration of $\mathrm{Pr}^{3+}$. The active subspace is highlighted, namely, $3 d$ and $4 f$, showing also the frozen electron occupation schemes that represent the two configurations at hand. Note the observed change of the electronic structure from $4 f^{2}$ to $3 d^{9} 4 f^{3}$ : the overall stabilization of the atomic orbital energies is induced by the creation of a core-hole in the $\operatorname{Pr} 3 d$ orbitals and the presence of additional electrons in the $4 f$ (Fig. 1).

The DFT calculations are performed by means of the ADF program package. ${ }^{11-13}$ The main results reported in this presentation are obtained using the generalized gradient approximation (GGA) functional following the PerdewBurke-Ernzerhof (PBE) parameterization. ${ }^{22}$ However, two additional DFT functionals are also tested including the local density approximation (LDA) Slater exchange and VoskoWilk-Nusair correlation (SVWN) ${ }^{23}$ and hybrid B3LYP. ${ }^{24}$ The molecular orbitals are expanded using multiple-zeta Slatertype orbital (STO) functions with polarization extra functions, ${ }^{25}$ i.e., TZ2P for lanthanides and TZP for other elements. The SCF is set-up to take into account all electrons. The relativistic corrections are treated with the Zeroth-Order Regular Approximation (ZORA) of the dirac equation method. ${ }^{26,27}$ All electronic structure calculations are done at the scalar ZORA relativistic level of theory, and the spin-orbit coupling interaction is included through the use of the spin-orbit ZORA method. ${ }^{26,27}$

\section{B. Effective ligand-field Hamiltonian}

Along with the line of thoughts intrinsic to the AOC-type calculation in DFT, ${ }^{14-21}$ the concept of effective ligand-field Hamiltonian insures a multi-reference algorithm, ${ }^{28}$ where the configuration interaction within the active subspace is easily captured. The ligand-field calculation starts with the converged DFT electronic structure that is obtained using the methodology described in Sec. II A. Based on a ligand-field analysis of the energies and radial functions of the Kohn-Sham orbitals (Fig. 1), the regular ligand-field parameters are thus non-empirically derived. 
The effective Hamiltonian $\left(H_{\text {eff }}\right)$ is used to calculate the multiplet energy levels of configuration $3 d^{9} 4 f^{n+1}$,

$$
H_{e f f}\left|\psi_{i}\right\rangle=E_{i}\left|\psi_{i}\right\rangle
$$

where $E_{i}\left(i=1,2, \ldots, N_{\left(3 d^{9} 4 f^{n+1}\right)}\right)$ is the $i$ th eigenvalue; and $\psi_{i}$ is a multi-electronic eigenfunction.

The effective Hamiltonian,

$$
H_{\text {eff }}=H_{0}+H_{E R}+H_{S O}+H_{L F},
$$

is defined so that the most relevant electronic interactions involved in the XAS process is taken into account, inter alia, configuration average energy correction $\left(H_{0}\right)$, inter-electron repulsion $\left(H_{E R}\right)$, spin-orbit coupling $\left(H_{S O}\right)$, and ligand-field splitting $\left(H_{L F}\right) .45$ The electronic interactions can be factorized into two parts: (i) the so-called reduced matrix-element dependent upon parameters and (ii) coefficients inherent to the angular part that are specific to the spin- and orbital-momenta coupling for the configuration at hand. These coefficients can be obtained from tables, tensor-algebra, or numerical tools. ${ }^{29,30}$ In this work, they are expressed in the basis of single determinants of spin-orbitals that belong to the two open-shell configurations $3 d^{9} 4 f^{n+1}$; they are computed by means of Matlab programing and the Matlab parallel toolbox; and they are stored with binary files, which can be soon downloaded from the ADF suite of software. ${ }^{11-13}$

Table I lists the calculated dimension of the matrix elements of the effective Hamiltonian in terms of the constant $n$ [see Eq. (3)] together with typical lanthanide ions that possess the specific open-shell configuration,

$$
N\left(3 d^{9} 4 f^{n+1}\right)=\left(\begin{array}{c}
10 \\
9
\end{array}\right)\left(\begin{array}{c}
14 \\
n+1
\end{array}\right) .
$$

The minimum and maximum dimensions that are obtained in the present work equal $10 \times 10$ and $34320 \times 34320$, respectively, (see Table I).

\section{Parameterization scheme}

The following parameters are required for the calculation of the multiplet energies and ligand-field splitting of configuration $3 d^{9} 4 f^{n+1}$ :

(i) An energy gap $\Delta E_{a v}$, which is defined to represent the shift of the energy of the multiplet manifold of the configuration $3 d^{9} 4 f^{n+1}$ vis-à-vis a reference configuration that is the ground $4 f^{n}$. The parameter $\Delta E_{a v}$ is engulfed

TABLE I. Dimension of the matrix elements of the effective Hamiltonian that is constructed in the present theoretical model, together with selective lanthanide ions that belong to configuration $3 d^{9} 4 f^{n+1}$.

\begin{tabular}{lcc}
\hline \hline$n$ & $N\left(3 d^{9} 4 f^{n+1}\right)$ & Lanthanide ions \\
\hline 13 & 10 & $\mathrm{Tm}^{2+}, \mathrm{Yb}^{3+}, \mathrm{Lu}^{4+}$ \\
$0-12$ & 140 & $\mathrm{La}^{3+}, \mathrm{Ce}^{4+}-\mathrm{Tm}^{3+}, \mathrm{Yb}^{4+}$ \\
$1-11$ & 910 & $\mathrm{Ce}^{3+}, \mathrm{Pr}^{4+}-\mathrm{Er}^{3+}$ \\
$2-10$ & 3640 & $\mathrm{Pr}^{3+}-\mathrm{Ho}^{3+}, \mathrm{Er}^{4+}$ \\
$3-9$ & 10010 & $\mathrm{Nd}^{3+}-\mathrm{Dy}^{3+}, \mathrm{Ho}^{4+}$ \\
$4-8$ & 20020 & $\mathrm{Pm}^{3+}-\mathrm{Tb}^{3+}$ \\
$5-7$ & 30030 & $\mathrm{Sm}^{3+}-\mathrm{Eu}^{2+}, \mathrm{Gd}^{3+}$ \\
6 & 34320 & $\mathrm{Eu}^{3+}$ \\
\hline \hline
\end{tabular}

in the term $\left(H_{0}\right)$ in Eq. (2). This term represents an operator with zeroth-order contributions,

$$
H_{0}=I_{N\left(3 d^{9} 4 f^{n+1}\right)} \Delta E_{a v},
$$

that conveys the so-called configuration average energy $^{29}$ but also the kinetic energy background and nuclear-electron attraction obtained from DFT. $I_{N\left(3 d^{9} 4 f^{n+1}\right)}$ is an identity matrix of dimensions $N\left(3 d^{9} 4 f^{n+1}\right) \times N\left(3 d^{9} 4 f^{n+1}\right)$ assuming further that the matrix elements of the three other terms $H_{E R}, H_{S O}$, and $H_{L F}$ in Eq. (2) are traceless blocks, ${ }^{18,19}$

$$
\begin{aligned}
H_{E R}= & \sum_{k=2,4,6} F^{k}(4 f, 4 f) \cdot \varphi_{k}(4 f, 4 f) \\
& +\sum_{k=2,4} F^{k}(3 d, 4 f) \cdot \varphi_{k}(3 d, 4 f) \\
& +\sum_{k=1,3,5} G^{k}(3 d, 4 f) \cdot \omega_{k}(3 d, 4 f) .
\end{aligned}
$$

(ii) A set of Slater-Condon integrals $F^{k}$ and $G^{k}$, which represent the Coulomb and Exchange interactions, respectively. These parameters are involved in the term $\left(H_{E R}\right)$ in Eq. (2), which is a two-electron operator. This term originates from the central-field approximation and perturbation theory of Slater. ${ }^{31}$ It is defined in Eq. (5), where $\varphi$ and $\omega$ represent the angular coefficients proper to a specific configuration. ${ }^{31}$ The parameters $F^{k}$ and $G^{k}$ are, in the first instance, responsible for the split of the energy of the configuration $3 d^{9} 4 f^{n+1}$ into atomic multiplet levels.

(iii) Spin-orbit coupling constants $\zeta_{3 d}$ and $\zeta_{4 f}$, which originate from the relativistic effect. They express the spinorbit structure of the atomic multiplet energies using the term $\left(H_{S O}\right)$ in Eq. (2). This term is a one-electron operator,

$$
H_{S O}=\sum_{i} \zeta_{n_{i} l_{i}}\left(r_{i}\right)\left(l_{i} \cdot s_{i}\right),
$$

where the coefficient $l_{i} \cdot s_{i}$ describes the coupling of the angular momenta of the spin and orbit in terms of $3 d$ and $4 f$ electrons, respectively. ${ }^{29,30}$

(iv) A ligand-field potential, which is a block matrix with 12 $\times 12$ elements. $^{32}$ This block matrix results from direct perturbation of the $3 d$ (with $5 \times 5$ elements) and $4 f$ (with $7 \times 7$ elements) orbitals as well as a second order perturbation (with $7 \times 5$ elements) that allows mixing $3 d$ and $4 f$ by symmetry. ${ }^{32}$ The ligand-field potential operates in the term $\left(H_{L F}\right)$ in Eq. (2), which is a oneelectron operator. $\left(H_{L F}\right)$ is often expressed with respect to $\left(C_{q}^{(k)}\right)$,

$$
\begin{aligned}
H_{L F}= & \sum_{k=2,4} \sum_{q=-k}^{k} B_{q}^{k}(3 d, 3 d) C_{q}^{(k)} \\
& +\sum_{k=2,4,6} \sum_{q=-k}^{k} B_{q}^{k}(4 f, 4 f) C_{q}^{(k)} \\
& +\sum_{k=1,3,5} \sum_{q=-k}^{k} B_{q}^{k}(3 d, 4 f) C_{q}^{(k)}
\end{aligned}
$$


that represents the spherical harmonic tensor operator. ${ }^{33}$ The parameters $B_{q}^{k}$ [Eq. (7)] act as constants in front of the multipolar expansion of the ligand-field potential. $^{33}$

\section{Non-empirical calculation of the parameters}

The energy gap $\Delta E_{a v}$ is calculated as the difference between total DFT energies [Eq. (8)]. These total DFT energies include both the nuclear and electronic contributions, where the lanthanide ion has the configuration $3 d^{9} 4 f^{n+1}$ and the reference configuration that is $4 f^{n}$, respectively,

$$
\Delta E_{a v}=E^{D F T}\left(3 d^{9} 4 f^{n+1}\right)-E^{D F T}\left(4 f^{n}\right) .
$$

The Slater-Condon integrals $F^{k}$ and $G^{k}$ are determined using $\bar{R}_{3 d}$ and $\bar{R}_{4 f}$ that are the dominant part of the radial functions extracted from the active Kohn-Sham orbitals centered on the lanthanide ion [Eqs. (9)-(11)]. They are expanded in terms of multiple-zeta STO functions in ADF, ${ }^{1-13}$

$$
\begin{aligned}
F^{k}(4 f, 4 f) & =\int_{0}^{\infty} \int_{0}^{\infty} \frac{r_{<}^{k}}{r_{>}^{k+1}} \bar{R}_{4 f}^{2}\left(r_{1}\right) \bar{R}_{4 f}^{2}\left(r_{2}\right) r_{1}^{2} r_{2}^{2} d r_{1} d r_{2}, \\
F^{k}(3 d, 4 f) & =\int_{0}^{\infty} \int_{0}^{\infty} \frac{r_{<}^{k}}{r_{>}^{k+1}} \bar{R}_{3 d}^{2}\left(r_{1}\right) \bar{R}_{4 f}^{2}\left(r_{2}\right) r_{1}^{2} r_{2}^{2} d r_{1} d r_{2},
\end{aligned}
$$

$$
\begin{aligned}
& G^{k}(3 d, 4 f) \\
& \quad=\int_{0}^{\infty} \int_{0}^{\infty} \frac{r_{<}^{k}}{r_{>}^{k+1}} \bar{R}_{3 d}\left(r_{1}\right) \bar{R}_{4 f}\left(r_{2}\right) \bar{R}_{4 f}\left(r_{1}\right) \bar{R}_{3 d}\left(r_{2}\right) r_{1}^{2} r_{2}^{2} d r_{1} d r_{2} .
\end{aligned}
$$

In Eqs. (9)-(11), $r_{<}$and $r_{>}$are the lesser and the greater of the distances $r_{1}$ and $r_{2}$ of two electrons from the lanthanide center, respectively. ${ }^{29,30}$ It should be noted that by taking into account the ligand-field splitting, the degeneracies of the $3 d$ and $4 f$ orbitals are lifted according to the lanthanide and ligand coordination chemistry. Figure 2 shows a graphical representation of the radial functions of the $4 f$ orbitals

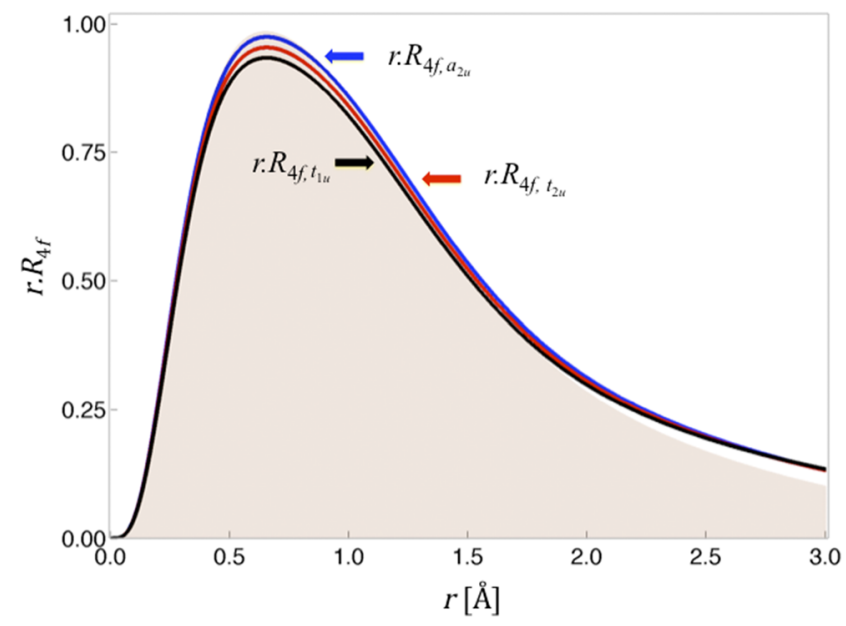

FIG. 2. Representation of the radial function of the Kohn-Sham orbitals with the dominant $4 f$ character of $\mathrm{Pr}^{3+}$ within configuration $3 d^{9} 4 f^{3}$, obtained by DFT using the GGA functional and scalar ZORA relativistic corrections, showing the different shapes $R_{4 f}$ for free ions (light-red area) and for molecular $\left(\mathrm{PrCl}_{6}\right)^{3-}$ clusters (red, blue, and black curves). of $\mathrm{Pr}^{3+}$ calculated in the isolated free ion and in octahedral hexachloro-praseodymium (III) ion (the $\mathrm{Pr}-\mathrm{Cl}$ bond length is set to $2.7735 \AA$ ). The impact of the chloride ligands on the radial functions is illustrated by the observation of three distinct profiles $R_{4 f, a_{2 u}}, R_{4 f, t_{1 u}}$, and $R_{4 f, t_{2 u}}$ (see Fig. 2) that indicate the splitting of the energy of $4 f$ orbitals into three components $a_{2 u}, t_{1 u}$, and $t_{2 u}$ named after the irreducible representations (irreps) of the $O_{h}$ point group.

Therefore it is convenient to define the terms $\bar{R}_{3 d}$ and $\bar{R}_{4 f}$ in Eqs. (9)-(11) as statistically averaged radial functions that are constructed by means of Eq. (12), i.e., by summing over all subsets $R_{3 d, i}$ and $R_{4 f, j}$ extracted from the DFT calculation,

$$
\bar{R}_{n l}(r)=\frac{1}{2 l+1} \sum_{i}^{2 l+1} R_{n l, i}(r) .
$$

It is also noteworthy to recall that five radial functions $\left(R_{3 d, i}\right)$ are obtained from the Kohn-Sham orbitals that predominantly possess lanthanide $3 d$ characters. Similarly, seven radial functions $\left(R_{4 f, j}\right)$ are obtained from the Kohn-Sham orbitals with $4 f$. Equation (12) complies with the fundamental concept of ligand-field theory, ${ }^{34,35}$ where electrons are moving in a central-field independent of the atomic $m l$ basis functions. ${ }^{34,35}$

Although the spin-orbit coupling constants $\zeta_{3 d}$ and $\zeta_{4 f}$ can also be obtained using the radial functions $\bar{R}_{3 d}$ and $\bar{R}_{4 f},{ }^{36-39}$ it is more convenient to take advantage of the spin-orbit ZORA method $^{26,27}$ in ADF. ${ }^{11-13}$ The primary advantage of using the ZORA method is that the spin-orbit coupling interaction is self-consistently treated. ${ }^{26,27,40}$ For atomic systems, the spinorbit coupling constants $\zeta_{3 d}$ and $\zeta_{4 f}$ can be directly derived from the eigenvalues of the ZORA Hamiltonian,

$$
\zeta_{n l}=\frac{2}{2 l+1}\left(\epsilon_{n l, j=l+\frac{1}{2}}^{Z O R A}-\epsilon_{n l, j=l-\frac{1}{2}}^{Z O R A}\right),
$$

where $\epsilon_{n l, j=l+\frac{1}{2}}^{Z O R A}$ and $\epsilon_{n l, j=l-\frac{1}{2}}^{Z O R A}$ are energies (see also Fig. 1), which belong to the atomic orbitals that have $n l$ characters, for instance $3 d$ and $4 f$. For a molecular system, a set of spin-orbit coupling constants can be also directly derived via the mathematical fitting procedure and symmetry analysis. $^{15}$

The parameters $B_{q}^{k}$ measure the strength of the interaction between the electrons of the lanthanide ion and the ligand coordination. They depend upon the coordination symmetry, i.e.,, they may exist or vanish according to the $k$ and $q$ indices. For instance, an octahedral coordination leads to three independent parameters such as $B_{0}^{4}(3 d, 3 d), B_{0}^{4}(4 f, 4 f)$, and $B_{0}^{6}(4 f, 4 f)$ that are non-zero by symmetry. ${ }^{32,41}$ These parameters are calculated from the energies of the Kohn-Sham orbitals,

$$
\begin{aligned}
& B_{0}^{4}(3 d, 3 d)=\frac{21}{10}\left(\epsilon_{3 d, e_{g}}-\epsilon_{3 d, t_{2 g}}\right), \\
& B_{0}^{4}(4 f, 4 f)=-\frac{39}{44}\left(3 \epsilon_{4 f, a_{2 u}}+4 \epsilon_{4 f, t_{2 u}}\right), \\
& B_{0}^{6}(4 f, 4 f)=\frac{36}{11(2+\sqrt{2})}\left(\epsilon_{4 f, a_{2 u}}+2 \epsilon_{4 f, t_{1 u}}\right)
\end{aligned}
$$

that have dominant $3 d\left(\epsilon_{3 d}\right)$ and $4 f\left(\epsilon_{4 f}\right)$ characters. The terms $e_{g}$ and $t_{2 g}$ denominate irreps of the $O_{h}$ point group that 
establish the splitting of the energy of the $3 d$ orbitals into two different molecular orbital levels, similar to the terms $a_{2 u}, t_{1 u}$, and $t_{2 u}$ described earlier. It is to be noted that Eqs. (15) and (16) work with the assumption that the term $B_{0}^{0}(4 f, 4 f)=0 .{ }^{32,38,39}$

\section{RESULTS AND DISCUSSION}

\section{A. Calculated parameters and validation}

Table II lists tabulated values of the energy gap $\Delta E_{a v}$, Slater-Condon integrals $F^{k}(4 f, 4 f), G^{k}(3 d, 4 f), F^{k}(3 d, 4 f)$, and spin-orbit coupling constants $\zeta_{3 d}$ and $\zeta_{4 f}$ for the trivalent oxidation state of all elements from La to $\mathrm{Yb}$. The parameters were calculated for configuration $3 d^{9} 4 f^{n+1}$ of the element concerned. For comparison, Table II contains also the reference values taken from Ref. 8. The parameters generally increase from La to $\mathrm{Yb}$. This is because the $3 d$ and $4 f$ radial functions shrink along the lanthanide series, which is the factor that induces the periodic trend (see also Fig. S1 of the supplementary material).

The change of the DFT functional along with the parameter calculations is tested by listing also in Table II the results obtained with LDA and hybrid functionals. Comparing the calculated parameters for different DFT functionals, it is remarkable that LDA and GGA results differ by less than $5 \%$ in magnitude. Besides the use of the hybrid functional, which contains a portion of exact exchange, ${ }^{24}$ has led to a slight reduction of the values of both Slater-Condon and spin-orbit coupling parameters. Thereby the hybrid functional improves slightly the precision of the calculation if compared to the reference data (see Table II). However, it fails to lead to a good precision of the energy gap $\Delta E_{a v}$, where the energy deviation is represented by about $12 \mathrm{eV}$ over the LDA and GGA data (see Table II). Apart from the rest of the parameters, the energy gap $\Delta E_{a v}$ makes important contributions, in the way that an overestimation of it leads to exaggeration, a strong energy shift of the multiplet structure of configuration $3 d^{9} 4 f^{n+1}$.

Figure 3 shows the comparison between the calculated parameters and reference data for lanthanide ions from $\mathrm{La}$ to $\mathrm{Yb}$. $P E$ represents the percentage errors that are obtained for equivalent parameters. For convenience, only the results obtained with GGA are shown since the results with LDA and hybrid functionals present similar trends. Where $P E$ values are negative, the parameters are underestimated by the DFT calculation (and vice versa). The Slater-Condon integrals $F^{k}(4 f, 4 f), G^{k}(3 d, 4 f)$, and $F^{k}(3 d, 4 f)$ are somewhat overestimated, especially for the late lanthanide series (from $\mathrm{Gd}$ to $\mathrm{Yb}$ ). The maximum deviation that is obtained in the calculation represents $20 \%$ over the reference data. On the other hand, the spin-orbit coupling constants $\zeta_{n l}$ and energy gap $\Delta E_{a v}$ are qualitatively good with less than $5 \%$ deviation over the reference data.

It should be noted that the definition of the calculated parameters in Table II does not include scaling factors or empirical corrections. In Ref. 8, the parameters are adjusted to fit the experimental findings. But in the present results, the screenings of inter-electron repulsion and spin-orbit coupling interaction are not possible with isolated lanthanide ions.
Therefore the comparison of the calculated parameters and reference data is a qualitative picture, which shows that both are comparable in magnitude, thus allowing validation of the DFT results.

\section{B. Simulated x-ray absorption spectra}

It is assumed that the dipole approximation is adequate to resolve the whole spectral profiles of the lanthanide $\mathrm{M}_{4,5}$-edge X-ray absorption. ${ }^{8}$ The excitation energies $\left(\Delta E_{i}\right)$ and intensities $\left(I_{i}\right)$ of the intra-atomic $4 f^{n}+h v \rightarrow 3 d^{9} 4 f^{n+1}$ electron transitions are calculated as follows:

$$
\begin{aligned}
\Delta E_{i} & =E_{i}-E_{0}, \\
I_{i} & \cong\left|\left\langle\psi_{i}|\mu| \psi_{0}\right\rangle\right|^{2} \frac{\gamma_{i}}{\pi\left(\left(h \nu-\Delta E_{i}\right)^{2}+\gamma_{i}^{2}\right)},
\end{aligned}
$$

where the transition moment function $\left\langle\psi_{i}|\mu| \psi_{0}\right\rangle$, which is operated via the matrix elements of the electric-dipole moment operator $(\mu)$, involves final $\psi_{i}$ and initial $\psi_{0}$ electronic states. $\psi_{i}$, with $i=1,2, \ldots$ and $N\left(3 d^{9} 4 f^{n+1}\right)$, forms the basis of the whole multiplet manifold of configuration $3 d^{9} 4 f^{n+1}$, whereas $\psi_{0}$ corresponds to the ground state of the multiplet manifold of configuration $4 f^{n}$ without considering temperature effects. $E_{i}$ and $E_{0}$ are their respective energy eigenvalues. In Eq. (18), the Lorentzian line shape is used to broaden the transition probabilities with a constant half-width at the half of the maximum parameter in order to simulate the finite lifetime of the final X-ray electronic states. ${ }^{42}$

Table III lists the initial $\psi_{0}$ electronic states that are obtained from the DFT calculation for the isolated trivalent lanthanide ions. The quantum $J$ numbers that are associated with the ground state multiplet of configuration $4 f^{n}$ are tabulated together with the most important $L S$ components due to the intermediate and spin-orbit coupling effects, which allows mixing of $S$ values. The $\psi_{0}$ 's possess a predominantly unique character because the spin-orbit coupling interaction of the $4 f$ electrons is not strong enough (see Table III). The results are in line with the prediction of the ground state according to a simple application of Hund's rule. The calculated leading percentages corresponding to the $L S$ components are also comparable with the experimental values that are collected in the framework of the NIST spectral database for trivalent lanthanide ions. ${ }^{43,44}$

Figure 4 shows the calculated intensity versus excitation energy of the intra-atomic $4 f^{n} \rightarrow 3 d^{9} 4 f^{n+1}$ electron transitions for the trivalent oxidation state of all elements from $\mathrm{La}$ to $\mathrm{Yb}$. The depicted figures are used to represent the lanthanide $\mathrm{M}_{4,5}$-edge $\mathrm{X}$-ray absorption spectra of the isolated ions. Examining the $J$ designations of the final $\psi_{i}$ states, a general trend is observed. The final $\psi_{i}$ states that possess non-zero transition probabilities always have values of $J$ similar or one unit upper or one unit lower than that of the corresponding initial $\psi_{0}$ state. It is well known that in the present context, the electric-dipole transitions between initial and final states obey $\Delta J=-1, \Delta J=0$, and $\Delta J=+1$ selection rules. ${ }^{8}$ It becomes therefore evident to discriminate separately the contributions of these three term selection rules in Fig. 4. In fact, of the three terms of the selection rules, the shape of the X-ray absorption spectrum can be easily decomposed. 
TABLE II. Energy gap parameter, Slater-Condon integrals, and spin-orbit coupling constants (in eV) for configuration $3 d^{9} 4 f^{n+1}$ of lanthanide ions obtained from DFT using LDA (a), GGA (b), and hybrid (c) functionals together with reference literature data (d).

\begin{tabular}{|c|c|c|c|c|c|c|c|c|}
\hline$n$ & & $\begin{array}{c}\mathrm{La}^{3+} \\
0\end{array}$ & $\begin{array}{c}\mathrm{Ce}^{3+} \\
1\end{array}$ & $\begin{array}{c}\mathrm{Pr}^{3+} \\
2\end{array}$ & $\begin{array}{c}\mathrm{Nd}^{3+} \\
3\end{array}$ & $\begin{array}{c}\mathrm{Pm}^{3+} \\
4\end{array}$ & $\begin{array}{c}\mathrm{Sm}^{3+} \\
5\end{array}$ & $\begin{array}{c}\mathrm{Eu}^{3+} \\
6\end{array}$ \\
\hline \multirow[t]{4}{*}{$\Delta \mathrm{E}_{a v}$} & (a) & 835.95 & 883.82 & 932.54 & 982.27 & 1033.2 & 1085.1 & 1137.9 \\
\hline & (b) & 835.96 & 883.86 & 932.61 & 982.36 & 1033.3 & 1085.2 & 1138.1 \\
\hline & (c) & 848.46 & 896.55 & 945.42 & 995.22 & 1046.2 & 1098.0 & 1150.8 \\
\hline & (d) & 838.10 & 886.40 & 934.20 & 983.90 & 1034.6 & 1086.0 & 1138.5 \\
\hline \multirow[t]{4}{*}{$F^{2}(4 f, 4 f)$} & (a) & $\ldots$ & 11.765 & 11.749 & 12.330 & 12.887 & 13.388 & 13.849 \\
\hline & (b) & $\ldots$ & 11.730 & 11.723 & 12.306 & 12.866 & 13.370 & 13.833 \\
\hline & (c) & $\ldots$ & 11.438 & 11.493 & 12.130 & 12.735 & 13.273 & 13.769 \\
\hline & (d) & $\ldots$ & 10.01 & 10.48 & 10.84 & 11.18 & 11.51 & 11.84 \\
\hline \multirow[t]{4}{*}{$F^{4}(4 f, 4 f)$} & (a) & $\ldots$ & 7.367 & 7.358 & 7.720 & 8.070 & 8.387 & 8.675 \\
\hline & (b) & $\ldots$ & 7.343 & 7.340 & 7.704 & 8.056 & 8.375 & 8.665 \\
\hline & (c) & $\ldots$ & 7.152 & 7.190 & 7.589 & 7.971 & 8.312 & 8.623 \\
\hline & (d) & $\ldots$ & 6.35 & 6.59 & 6.81 & 7.03 & 7.24 & 7.44 \\
\hline \multirow[t]{4}{*}{$F^{6}(4 f, 4 f)$} & (a) & $\ldots$ & 5.295 & 5.289 & 5.548 & 5.801 & 6.029 & 6.237 \\
\hline & (b) & $\ldots$ & 5.277 & 5.276 & 5.537 & 5.791 & 6.021 & 6.229 \\
\hline & (c) & $\ldots$ & 5.137 & 5.166 & 5.453 & 5.728 & 5.975 & 6.199 \\
\hline & (d) & $\ldots$ & 4.57 & 4.74 & 4.91 & 5.07 & 5.21 & 5.36 \\
\hline \multirow[t]{4}{*}{$G^{1}(3 d, 4 f)$} & (a) & 4.089 & 4.495 & 4.779 & 5.175 & 5.491 & 5.787 & 6.108 \\
\hline & (b) & 4.063 & 4.468 & 4.753 & 5.147 & 5.463 & 5.760 & 6.082 \\
\hline & (c) & 3.873 & 4.308 & 4.615 & 5.029 & 5.365 & 5.678 & 6.014 \\
\hline & (d) & 3.78 & 4.06 & 4.33 & 4.59 & 4.84 & 5.09 & 5.33 \\
\hline \multirow[t]{4}{*}{$G^{3}(3 d, 4 f)$} & (a) & 2.421 & 2.662 & 2.829 & 3.063 & 3.252 & 3.428 & 3.618 \\
\hline & (b) & 2.406 & 2.646 & 2.814 & 3.047 & 3.236 & 3.412 & 3.603 \\
\hline & (c) & 2.293 & 2.551 & 2.732 & 2.977 & 3.177 & 3.363 & 3.562 \\
\hline & (d) & 2.21 & 2.37 & 2.53 & 2.69 & 2.83 & 2.98 & 3.12 \\
\hline \multirow[t]{4}{*}{$G^{5}(3 d, 4 f)$} & (a) & 1.680 & 1.847 & 1.963 & 2.125 & 2.256 & 2.378 & 2.510 \\
\hline & (b) & 1.669 & 1.836 & 1.952 & 2.113 & 2.245 & 2.367 & 2.499 \\
\hline & (c) & 1.591 & 1.770 & 1.895 & 2.065 & 2.204 & 2.333 & 2.471 \\
\hline & (d) & 1.52 & 1.64 & 1.75 & 1.85 & 1.96 & 2.06 & 2.16 \\
\hline \multirow[t]{4}{*}{$F^{2}(3 d, 4 f)$} & (a) & 6.508 & 7.003 & 7.295 & 7.754 & 8.159 & 8.537 & 8.919 \\
\hline & (b) & 6.478 & 6.974 & 7.267 & 7.726 & 8.132 & 8.512 & 8.894 \\
\hline & (c) & 6.234 & 6.776 & 7.101 & 7.589 & 8.020 & 8.421 & 8.822 \\
\hline & (d) & 5.65 & 5.99 & 6.31 & 6.62 & 6.92 & 7.21 & 7.50 \\
\hline \multirow[t]{4}{*}{$F^{4}(3 d, 4 f)$} & (a) & 2.874 & 3.127 & 3.290 & 3.530 & 3.733 & 3.923 & 4.120 \\
\hline & (b) & 2.858 & 3.111 & 3.275 & 3.514 & 3.717 & 3.908 & 4.105 \\
\hline & (c) & 2.735 & 3.009 & 3.188 & 3.440 & 3.657 & 3.858 & 4.064 \\
\hline & (d) & 2.53 & 2.71 & 2.87 & 3.03 & 3.19 & 3.34 & 3.48 \\
\hline \multirow[t]{4}{*}{$\zeta_{3 d}$} & (a) & 6.882 & 7.527 & 8.218 & 8.960 & 9.750 & 10.585 & 11.473 \\
\hline & (b) & 6.886 & 7.531 & 8.222 & 8.964 & 9.756 & 10.590 & 11.478 \\
\hline & (c) & 6.936 & 7.584 & 8.279 & 9.024 & 9.819 & 10.657 & 11.549 \\
\hline & (d) & 6.80 & 7.45 & 8.14 & 8.88 & 9.67 & 10.51 & 11.41 \\
\hline \multirow[t]{5}{*}{$\xi_{4 f}$} & (a) & 0.086 & 0.102 & 0.119 & 0.138 & 0.156 & 0.176 & 0.198 \\
\hline & (b) & 0.086 & 0.101 & 0.119 & 0.137 & 0.155 & 0.175 & 0.197 \\
\hline & (c) & 0.081 & 0.097 & 0.115 & 0.135 & 0.154 & 0.175 & 0.198 \\
\hline & (d) & 0.088 & 0.106 & 0.118 & 0.130 & 0.160 & 0.180 & 0.200 \\
\hline & & $\mathrm{Gd}^{3+}$ & $\mathrm{Tb}^{3+}$ & $\mathrm{Dy}^{3+}$ & $\mathrm{Ho}^{3+}$ & $\mathrm{Er}^{3+}$ & $\mathrm{Tm}^{3+}$ & $\mathrm{Yb}^{3+}$ \\
\hline$n$ & & 7 & 8 & 9 & 10 & 11 & 12 & 13 \\
\hline \multirow[t]{4}{*}{$\Delta \mathrm{E}_{a v}$} & (a) & 1191.9 & 1247.1 & 1303.2 & 1360.6 & 1419.2 & 1478.8 & 1539.7 \\
\hline & (b) & 1192.1 & 1247.3 & 1303.4 & 1360.7 & 1419.4 & 1478.9 & 1539.8 \\
\hline & (c) & 1204.6 & 1259.6 & 1315.5 & 1372.7 & 1431.0 & 1490.2 & 1550.7 \\
\hline & (d) & 1192.9 & 1247.8 & 1303.1 & 1359.7 & 1418.4 & 1478.9 & 1538.3 \\
\hline \multirow[t]{4}{*}{$F^{2}(4 f, 4 f)$} & (a) & 14.567 & 14.625 & 15.035 & 15.450 & 15.842 & $\ldots$ & $\ldots$ \\
\hline & (b) & 14.551 & 14.617 & 15.028 & 15.445 & 15.838 & $\ldots$ & $\ldots$ \\
\hline & (c) & 14.512 & 14.613 & 15.052 & 15.496 & 15.914 & $\ldots$ & $\ldots$ \\
\hline & (d) & 12.16 & 12.47 & 12.77 & 13.07 & 13.37 & $\ldots$ & $\ldots$ \\
\hline \multirow[t]{2}{*}{$F^{4}(4 f, 4 f)$} & (a) & 9.122 & 9.156 & 9.411 & 9.672 & 9.918 & $\ldots$ & $\ldots$ \\
\hline & (b) & 9.113 & 9.151 & 9.407 & 9.669 & 9.916 & $\ldots$ & $\ldots$ \\
\hline
\end{tabular}


TABLE II. (Continued.)

\begin{tabular}{|c|c|c|c|c|c|c|c|c|}
\hline$n$ & & $\begin{array}{c}\mathrm{Gd}^{3+} \\
7\end{array}$ & $\begin{array}{c}\mathrm{Tb}^{3+} \\
8\end{array}$ & $\begin{array}{c}\mathrm{Dy}^{3+} \\
9\end{array}$ & $\begin{array}{c}\mathrm{Ho}^{3+} \\
10\end{array}$ & $\begin{array}{c}\mathrm{Er}^{3+} \\
11\end{array}$ & $\begin{array}{c}\mathrm{Tm}^{3+} \\
12\end{array}$ & $\begin{array}{c}\mathrm{Yb}^{3+} \\
13\end{array}$ \\
\hline \multirow{6}{*}{$F^{6}(4 f, 4 f)$} & (c) & 9.088 & 9.150 & 9.424 & 9.705 & 9.968 & $\ldots$ & $\ldots$ \\
\hline & (d) & 7.64 & 7.84 & 8.03 & 8.21 & 8.40 & $\ldots$ & $\ldots$ \\
\hline & (a) & 6.557 & 6.581 & 6.763 & 6.952 & 7.129 & $\ldots$ & $\ldots$ \\
\hline & (b) & 6.550 & 6.577 & 6.760 & 6.950 & 7.127 & $\ldots$ & $\ldots$ \\
\hline & (c) & 6.533 & 6.577 & 6.774 & 6.976 & 7.166 & $\ldots$ & $\ldots$ \\
\hline & (d) & 5.50 & 5.64 & 5.78 & 5.91 & 6.04 & $\ldots$ & $\ldots$ \\
\hline \multirow[t]{4}{*}{$G^{1}(3 d, 4 f)$} & (a) & 6.470 & 6.663 & 6.949 & 7.212 & 7.449 & 7.722 & $\ldots$ \\
\hline & (b) & 6.444 & 6.638 & 6.925 & 7.189 & 7.427 & 7.700 & $\ldots$ \\
\hline & (c) & 6.389 & 6.600 & 6.900 & 7.176 & 7.427 & 7.713 & $\ldots$ \\
\hline & (d) & 5.56 & 5.79 & 6.02 & 6.24 & 6.46 & 6.68 & $\ldots$ \\
\hline \multirow[t]{4}{*}{$G^{3}(3 d, 4 f)$} & (a) & 3.833 & 3.949 & 4.119 & 4.275 & 4.417 & 4.580 & $\ldots$ \\
\hline & (b) & 3.817 & 3.934 & 4.104 & 4.261 & 4.404 & 4.567 & $\ldots$ \\
\hline & (c) & 3.785 & 3.910 & 4.089 & 4.253 & 4.403 & 4.573 & $\ldots$ \\
\hline & (d) & 3.26 & 3.40 & 3.53 & 3.66 & 3.79 & 3.92 & $\ldots$ \\
\hline \multirow[t]{4}{*}{$G^{5}(3 d, 4 f)$} & (a) & 2.659 & 2.740 & 2.858 & 2.967 & 3.066 & 3.179 & $\ldots$ \\
\hline & (b) & 2.649 & 2.730 & 2.848 & 2.958 & 3.057 & 3.170 & $\ldots$ \\
\hline & (c) & 2.626 & 2.713 & 2.837 & 2.952 & 3.056 & 3.174 & $\ldots$ \\
\hline & (d) & 2.25 & 3.35 & 2.44 & 2.53 & 2.62 & 2.71 & $\ldots$ \\
\hline \multirow[t]{4}{*}{$F^{2}(3 d, 4 f)$} & (a) & 9.368 & 9.590 & 9.933 & 10.264 & 10.573 & 10.898 & $\ldots$ \\
\hline & (b) & 9.344 & 9.568 & 9.912 & 10.245 & 10.555 & 10.880 & $\ldots$ \\
\hline & (c) & 9.288 & 9.532 & 9.892 & 10.240 & 10.565 & 10.905 & $\ldots$ \\
\hline & (d) & 7.77 & 8.04 & 8.31 & 8.57 & 8.83 & 9.09 & $\ldots$ \\
\hline \multirow[t]{4}{*}{$F^{4}(3 d, 4 f)$} & (a) & 4.347 & 4.465 & 4.642 & 4.810 & 4.964 & 5.133 & $\ldots$ \\
\hline & (b) & 4.332 & 4.452 & 4.629 & 4.798 & 4.953 & 5.121 & $\ldots$ \\
\hline & (c) & 4.300 & 4.430 & 4.430 & 4.615 & 4.792 & 5.130 & $\ldots$ \\
\hline & (d) & 3.63 & 3.77 & 3.91 & 4.04 & 4.18 & 4.31 & $\ldots$ \\
\hline \multirow[t]{4}{*}{$\zeta_{3 d}$} & (a) & 12.413 & 13.418 & 14.473 & 15.600 & 16.781 & 18.036 & 19.352 \\
\hline & (b) & 12.418 & 13.424 & 14.479 & 15.606 & 16.787 & 18.043 & 19.359 \\
\hline & (c) & 12.493 & 13.503 & 14.562 & 15.694 & 16.880 & 18.141 & 19.462 \\
\hline & (d) & 12.36 & 13.37 & 14.44 & 15.57 & 16.78 & 18.05 & 19.39 \\
\hline \multirow[t]{4}{*}{$\zeta_{4 f}$} & (a) & 0.221 & 0.246 & 0.273 & 0.301 & 0.329 & 0.362 & $\ldots$ \\
\hline & (b) & 0.221 & 0.245 & 0.272 & 0.300 & 0.329 & 0.361 & $\ldots$ \\
\hline & (c) & 0.223 & 0.249 & 0.278 & 0.307 & 0.339 & 0.374 & $\ldots$ \\
\hline & (d) & 0.222 & 0.249 & 0.277 & 0.305 & 0.336 & 0.368 & $\ldots$ \\
\hline
\end{tabular}

For lanthanide ions with $J=0$ initial state, namely, $\mathrm{La}^{3+}$ and $\mathrm{Eu}^{3+}$, the calculated X-ray absorption spectra enclose only the contribution of $\Delta J=+1$ [see Figs. 4(a) and 4(g)].

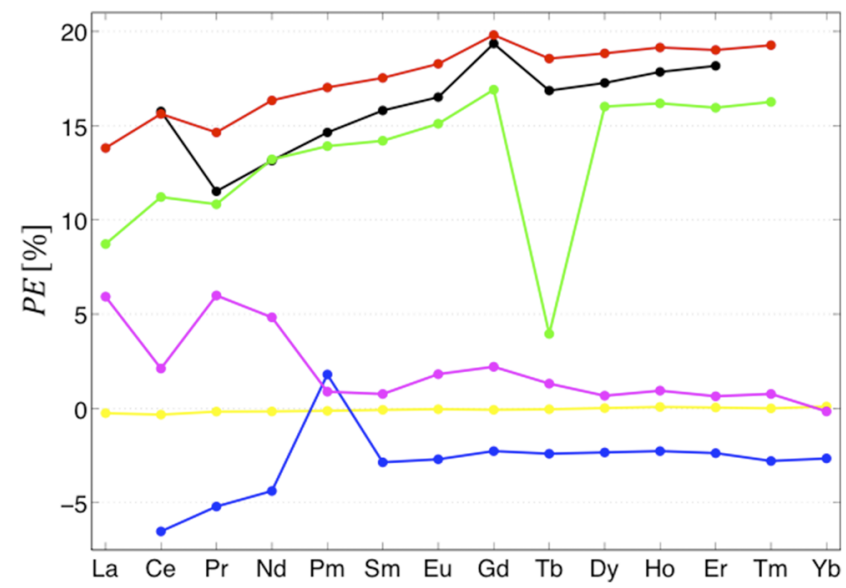

FIG. 3. Calculated percentage error $(P E)$ of the parameters with respect to reference data taken from the literature: $\Delta E_{a v}$ (in yellow), $\zeta_{4 f}$ (in blue), $\zeta_{3 d}$ (in magenta), $F^{k}(4 f, 4 f)$ (in black), $G^{k}(3 d, 4 f)$ (in green), and $F^{k}(3 d, 4 f)$ (in red).
On the other hand, for the $\mathrm{Yb}^{3+}$ ion, the calculated $\mathrm{X}$-ray absorption spectrum encloses only the contribution of $\Delta J=-1$ [see Fig. 4(n)]. In this case, the final electronic state belongs to configuration $3 d^{9} 4 f^{14}$. This configuration forms the basis of the spectral term ${ }^{2} \mathrm{D}$, which splits in energy into two components with $J=3 / 2$ and $J=5 / 2$, respectively. This latter one is permitted by the electric-dipole moment. For the $\mathrm{Tm}^{3+}$ ion, the calculated X-ray absorption spectrum does not have any contribution from $\Delta J=+1$ because the final electronic state with $J=7$ value is not possible for configuration $3 d^{9} 4 f^{13}$ [see Fig. 4(m)]. For the rest of the lanthanide ions, however, all the three denominated selection rules of the electric-dipole transitions contribute to the calculated spectral profiles.

For comparison, the experimental results that are previously reported by Thole et al. ${ }^{8}$ are also reproduced in Fig. 4 . The experimental spectra are not reproduced by the numerical scan of the pdf file support, but they are imitated by recalculating the $\mathrm{X}$-ray absorption spectra using the reference parameters listed in Table II. That is why, Fig. 4 encloses also a reference spectrum for the $\mathrm{Pm}^{3+}$ ion, also known as radioactive element. It is convenient to limit the comparative study to the 
TABLE III. Calculated electronic ground states of trivalent lanthanide ions within configuration $4 f^{n}$ that are used for the initial $\psi_{0}$ states: quantum $J$ number, multiplicity, and wavefunction (Eig.) in terms of leading percentages of atomic multiplet terms.

\begin{tabular}{lcccc}
\hline \hline & $n$ & $J^{\mathrm{a}}$ & Mult. & Eig. \\
\hline $\mathrm{La}^{3+}$ & 0 & 0 & 1 & $100 \%{ }^{1} \mathrm{~S}$ \\
$\mathrm{Ce}^{3+}$ & 1 & $5 / 2$ & 6 & $100 \%{ }^{2} \mathrm{~F}$ \\
$\mathrm{Pr}^{3+}$ & 2 & 4 & 9 & $97.0 \%{ }^{3} \mathrm{H}+2.9 \%{ }^{1} \mathrm{G}$ \\
$\mathrm{Nd}^{3+}$ & 3 & $9 / 2$ & 10 & $97.3 \%{ }^{4} \mathrm{I}+2.7 \%{ }^{2} \mathrm{H}$ \\
$\mathrm{Pm}^{3+}$ & 4 & 4 & 9 & $97.7 \%{ }^{5} \mathrm{I}+2.3 \%{ }^{3} \mathrm{H}$ \\
$\mathrm{Sm}^{3+}$ & 5 & $5 / 2$ & 6 & $96.3 \%{ }^{6} \mathrm{H}+3.2 \%{ }^{4} \mathrm{G}$ \\
$\mathrm{Eu}^{3+}$ & 6 & 0 & 1 & $93.8 \%{ }^{7} \mathrm{~F}+5.6 \%{ }^{5} \mathrm{D}$ \\
$\mathrm{Gd}^{3+}$ & 7 & $7 / 2$ & 8 & $97.6 \%{ }^{8} \mathrm{~S}+2.3 \%{ }^{6} \mathrm{P}$ \\
$\mathrm{Tb}^{3+}$ & 8 & 6 & 13 & $96.0 \%{ }^{7} \mathrm{~F}+3.6 \%{ }^{5} \mathrm{G}$ \\
$\mathrm{Dy}^{3+}$ & 9 & $15 / 2$ & 16 & $94.2 \%{ }^{6} \mathrm{H}+5.5 \%{ }^{4} \mathrm{I}$ \\
$\mathrm{Ho}^{3+}$ & 10 & 8 & 17 & $93.9 \%{ }^{5} \mathrm{I}+6.0 \%{ }^{3} \mathrm{~K}$ \\
$\mathrm{Er}^{3+}$ & 11 & $15 / 2$ & 16 & $97.5 \%{ }^{4} \mathrm{I}+2.4 \%{ }^{2} \mathrm{~K}$ \\
$\mathrm{Tm}^{3+}$ & 12 & 6 & 13 & $99.3 \%{ }^{3} \mathrm{H}+0.7 \%{ }^{1} \mathrm{I}$ \\
$\mathrm{Yb}^{3+}$ & 13 & $7 / 2$ & 8 & $100 \%{ }^{2} \mathrm{~F}$ \\
\hline \hline
\end{tabular}

a The multiplet energies of configuration $4 f^{n}$ are solved using a similar procedure as the one described in Sec. II. The parameters $F^{k}(4 f, 4 f)$ and $\zeta_{4 f}$ are left. Their calculated values are listed in Table S1 of the supplementary material.

excitation energies and intensities only, without addressing issues related to core-hole and experimental broadening effects and their influence on the spectral profiles. Indeed, these points are not easily tractable by the pure first principles studies and require joint experimental studies with multiple techniques. ${ }^{45}$ Therefore, a Lorentzian function with a constant half-width at the half of the maximum parameter, i.e., $\gamma_{i}=\gamma=0.4 \mathrm{eV}$ is also used to broaden the reference data for all elements from La to $\mathrm{Yb}$.

Both the theoretical and reference spectra in Fig. 4 present very similar features that are primarily distinguished by the two strong absorption bands due to the large spin-orbit coupling constant of the lanthanide $3 d$ electrons. The theoretical and the reference spectra contain also fine structures that arise from the multiplet energy levels of the configuration $3 d^{9} 4 f^{n+1}$ of the lanthanide ion. The discrepancies between the theoretical and the reference spectra are mainly found in the excitation energies $(\Delta E)$, where slight energy shifts are observed in the DFT calculation. The energy shifts that represent less than $5 \mathrm{eV}$ can be seen in the spectra of $\mathrm{La}^{3+}$ [Fig. 4(a)], $\mathrm{Ce}^{3+}$ [Fig. 4(b)], $\mathrm{Pr}^{3+}$ [Fig. 4(c)], $\mathrm{Nd}^{3+}$ [Fig. 4(d)], and $\mathrm{Pm}^{3+}$ [Fig. 4(e)].

\section{Case study: Lanthanide elpasolite structures}

The effects of the chemical ligand coordination on the spectral profiles are analyzed in the following by the study of the solid state $\mathrm{Cs}_{2} \mathrm{NaPrX}_{6}$ compounds, with $\mathrm{X}=\mathrm{F}, \mathrm{Cl}$, and $\mathrm{Br}$. The advantage of treating these compounds lies in fact that their crystal structures present the elpasolite structure type, ${ }^{46-48}$ giving rise to $\operatorname{Pr}$ coordination with $O_{h}$ symmetry.

It should be noted that the present theoretical model cannot deal with periodical crystal structures. Therefore it is convenient to construct a realistic molecular cluster model, from which the geometry optimization, multiplet energy calculation, and X-ray spectra simulation are performed. A positively charged molecular cluster containing the atomic coordinates of the praseodymium ion plus the six $X$ ligands together with the next nearest neighbor formed by six sodium atoms is chosen as a prototype that appropriately represents the local atomic environment around the lanthanide center. This molecular cluster is selectively represented in Fig. 5 by balls and sticks, together with the rest of crystal lattice of $\mathrm{Cs}_{2} \mathrm{NaPrX}_{6}$. In addition to the molecular cluster, a set of point charges is also included that allows simulation of the Madelung potential representing the long-range interaction due to the periodicity of $\mathrm{Cs}_{2} \mathrm{NaPrX}_{6}$. The symmetry of the ensemble is confined to the octahedral $\left(O_{h}\right)$ point group.

For the geometry optimization, the coordinates of the molecular cluster are relaxed having two degrees of freedom, namely, the distances $\operatorname{Pr}-\mathrm{X}\left(d_{P r-X}\right)$ and $\mathrm{X}-\mathrm{Na}\left(d_{X-N a}\right)$, with $\mathrm{X}=\mathrm{F}, \mathrm{Cl}$, and $\mathrm{Br}$. The electronic structures of the $\mathrm{Pr}$ ion is frozen, having an AOC scheme that represents the ground configuration $4 f^{2}$ because the smearing of valence electrons on the whole manifold of the lanthanide $4 f$ orbitals favors the octahedral symmetry. The calculated geometrical parameters $d_{P r-X}$ are $2.3232 \AA, 2.7735 \AA$, and $2.9227 \AA$, respectively, for $\mathrm{X}=\mathrm{F}$, $\mathrm{Cl}$, and $\mathrm{Br}$. These optimized bond lengths are in good agreement with values reported in the literature, ${ }^{50-52}$ but slightly smaller than the sum of ionic radii of $\operatorname{Pr}^{3+}$ and $\mathrm{X}^{-}$ligands in the $O_{h}$ coordination. ${ }^{53}$

The energy diagrams for the Kohn-Sham orbitals obtained for the molecular clusters are given in Fig. S16 of the supplementary material. The present DFT calculation procedure gives a quantitative picture of the ligand-field and bonding regime acting on the $\operatorname{Pr}^{3+}$ ion. The results show that three Kohn-Sham orbitals that belong to the $a_{2 u}, t_{1 u}$, and $t_{2 u}$ irreps of the $O_{h}$ point group possess dominant $\operatorname{Pr} 4 f$ characters. The energy order follows $\varepsilon_{a_{2 u}}<\varepsilon_{t_{2 u}}<\varepsilon_{t_{1 u}}$ independent of the ligand systems (see Fig. S16). The $a_{2 u}$ orbital, with dominant $\operatorname{Pr} 4 f_{x y z}$, is less perturbed because it has, in terms of covalence, the weakest Pr-ligand overlap integral, in opposition to the three-fold degenerate $t_{1 u}$ orbitals, as per the conventional octahedral ligand-field picture. The energy separation between the $a_{2 u}$ and $t_{1 u}$ orbitals $\left(\varepsilon_{t_{1 u}}-\varepsilon_{a_{2 u}}\right)$ varies upon the nature of the ligand coordination. It decreases in the order $\mathrm{F}^{-}>\mathrm{Cl}^{-}$ $>\mathrm{Br}^{-}$, in line with the so-called spectro-chemical series of ligands.

The octahedral ligand-field splits the energy of the ground state atomic multiplet of $\operatorname{Pr}^{3+}$, i.e., the term assimilated with $J=4$ in Table III that arises from configuration $4 f^{2}$. It is obtained one singlet $\mathrm{A}_{1 \mathrm{~g}}$, one doublet $\mathrm{E}_{\mathrm{g}}$, and two triplets $\mathrm{T}_{1 \mathrm{~g}}$ and $\mathrm{T}_{2 \mathrm{~g}}$ states. Table IV lists the calculated energies of these terms together with experimental reference data taken from the literature ${ }^{47-49}$ for comparison. The results show that the ground state of $\operatorname{Pr}^{3+}$ in the present elpasolite crystals is the single degenerate $\mathrm{A}_{1 \mathrm{~g}}$ regardless of the ligand systems. The $\mathrm{T}_{1 \mathrm{~g}}, \mathrm{E}_{\mathrm{g}}$, and $\mathrm{T}_{2 \mathrm{~g}}$ represent the first, second, and third excited states, respectively, whose energy separation vis-à-vis the ground state increases in the series $\left(\mathrm{PrBr}_{6}\right)^{3-}<\left(\mathrm{PrCl}_{6}\right)^{3-}$ $<\left(\mathrm{PrF}_{6}\right)^{3-}$. The agreement with the experimental data ${ }^{47-49}$ is relatively good, as it can be seen in Table IV.

Table $\mathrm{V}$ lists tabulated values of the energy gap $\Delta \mathrm{E}_{a v}$, Slater-Condon integrals $F^{k}(4 f, 4 f), G^{k}(3 d, 4 f), F^{k}(3 d, 4 f)$, spin-orbit coupling constants $\zeta_{3 d}$ and $\zeta_{4 f}$ and ligand-field parameters $B_{q}^{k}(3 d, 3 d)$ and $B_{q}^{k}(4 f, 4 f)$ for $\operatorname{Pr}^{3+}$ in the present 

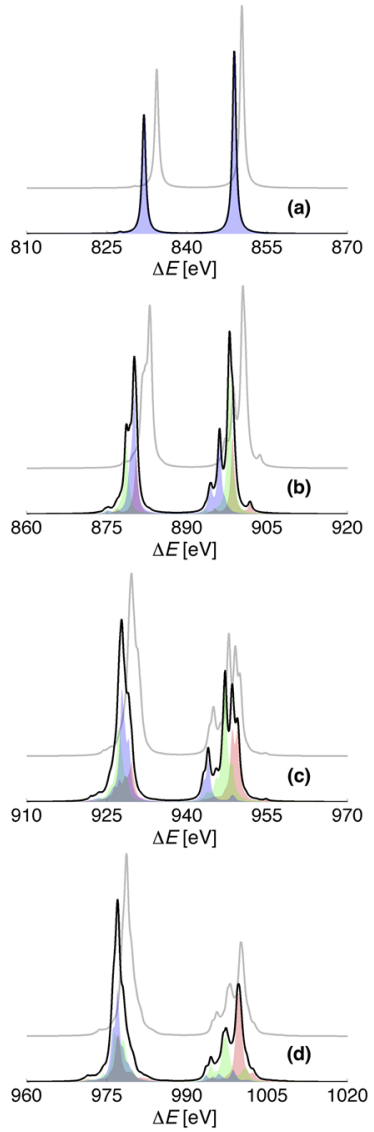
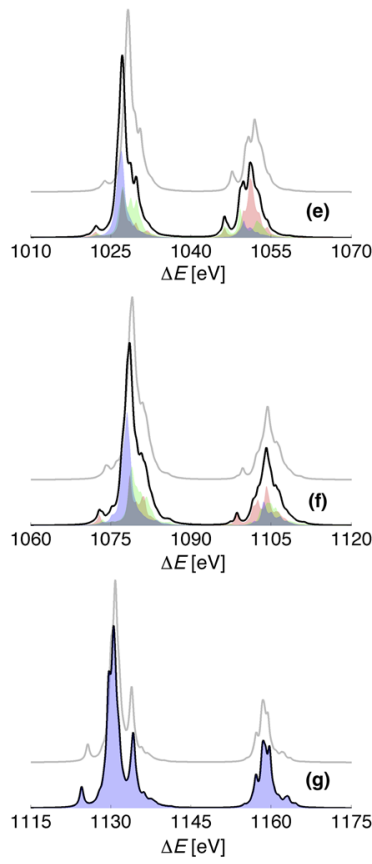

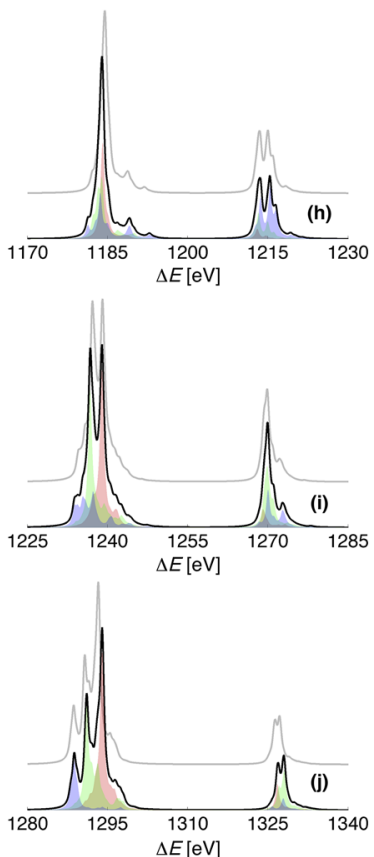

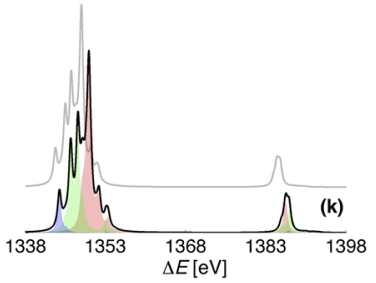
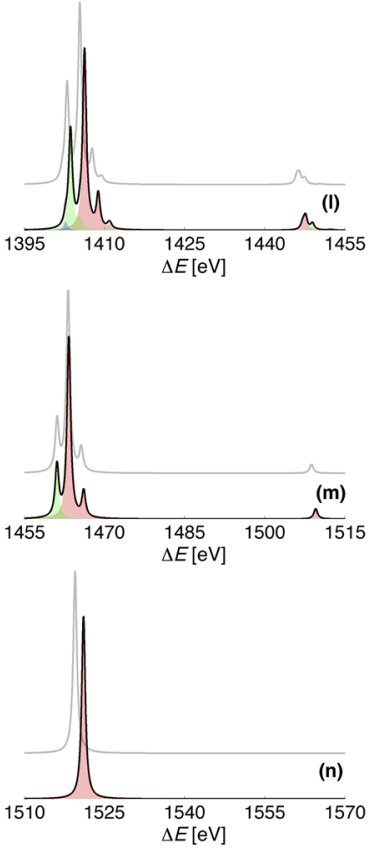

FIG. 4. Calculated spectra of lanthanide $\mathrm{M}_{4,5}$-edge X-ray absorption of $\mathrm{La}^{3+}(\mathrm{a}), \mathrm{Ce}^{3+}$ (b), $\mathrm{Pr}^{3+}(\mathrm{c}), \mathrm{Nd}^{3+}(\mathrm{d}), \mathrm{Pm}^{3+}(\mathrm{e}), \mathrm{Sm}^{3+}(\mathrm{f}), \mathrm{Eu}^{3+}(\mathrm{g}), \mathrm{Gd}^{3+}(\mathrm{h}), \mathrm{Tb}^{3+}(\mathrm{i})$, $\mathrm{Dy}^{3+}(\mathrm{j}), \mathrm{Ho}^{3+}(\mathrm{k}), \mathrm{Er}^{3+}(\mathrm{l}), \mathrm{Tm}^{3+}(\mathrm{m})$, and $\mathrm{Yb}^{3+}(\mathrm{n})$ showing the calculated intensity versus excitation energies of the intra-atomic $4 f^{n} \rightarrow 3 d^{9} 4 f^{n+1}$ electron transitions. For each subplot depicted in the figure, the $x$-coordinate (abscissa) represents the calculated excitation energies $(\Delta E)$ and the $y$-coordinate (ordinate) represents the convoluted calculated intensities ( $I$ ) (black curves) that are shown in arbitrary units. A Lorentzian function is used to broaden the transition probabilities taking a constant half-width at the half of the maximum parameter, $\gamma_{i}=\gamma=0.4 \mathrm{eV}$ for the all manifolds of the multiplet levels of configuration $3 d^{9} 4 f^{n+1}$. For clarity, the results that are previously reported in Ref. 8 are reproduced (gray curves) allowing a schematic comparison between the theoretical and reference spectra. The whole manifold of the multiplet levels of configuration $3 d^{9} 4 f^{n+1}$ contributes to the intensity calculations following the electric-dipole moment approximation, i.e., each final X-ray electronic state that has $\Delta J=-1, \Delta J=0$, and $\Delta J=+1$ values with respect to the initial state in Table III possess non-zero transition probabilities. Their contributions to the total spectrum are represented with the red-, green-, and blue-colored areas for $\Delta J=-1, \Delta J=0$, and $\Delta J=+1$, respectively. High quality figures are available in Figs. S2-S15 of the supplementary material.

elpasolite structures. The parameters were calculated for configuration $3 d^{9} 4 f^{3}$ of $\mathrm{Pr}^{3+}$. The parameters are compared to the results that are previously listed in Table II for the isolated

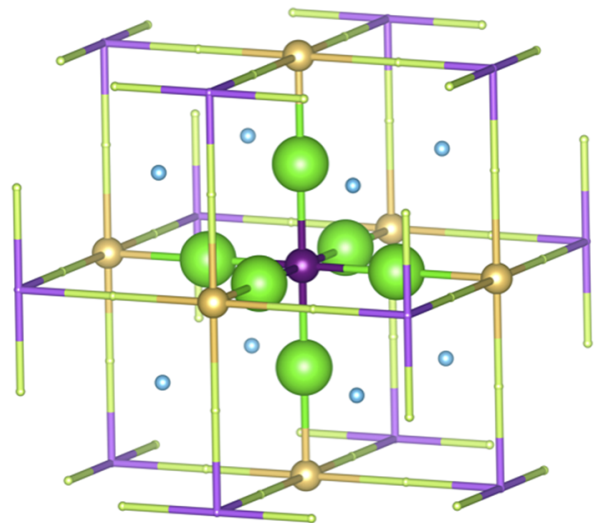

FIG. 5. Local atomic structure of the $\mathrm{Pr}^{3+}$ ion in $\mathrm{Cs}_{2} \mathrm{NaPrX}_{6}$, with $\mathrm{X}=\mathrm{F}, \mathrm{Cl}$, and $\mathrm{Br}$, crystallizing in the elpasolite structure type. Color code: $\operatorname{Pr}$ (violet), $\mathrm{X}$ (green), $\mathrm{Na}$ (yellow), and Cs (blue).
$\mathrm{Pr}^{3+}$ ion system. In Table $\mathrm{V}$, the quantity $\beta$ expresses the ratio between calculated parameters in the molecular cluster and in the free ion.

The change of the Slater-Condon integrals from free ion to molecular systems results from the different shape radial

TABLE IV. Calculated energy levels (in meV) of the ground state multiplet of the $\mathrm{Pr}^{3+}$ ion within configuration $4 f^{2}$ in the present elpasolite crystals, obtained from the DFT calculation using GGA functional, together with some experimental values.

\begin{tabular}{lcccccccc}
\hline \hline & \multicolumn{2}{c}{$\left(\mathrm{PrF}_{6}\right)^{3-}$} & & \multicolumn{2}{c}{$\left(\mathrm{PrCl}_{6}\right)^{3-}$} & & \multicolumn{2}{c}{$\left(\mathrm{PrBr}_{6}\right)^{3-}$} \\
\cline { 2 - 3 } & Calc. $^{\mathrm{a}}$ & Expt. $^{\mathrm{b}}$ & & Calc. $^{\mathrm{a}}$ & Expt. $^{\mathrm{b}}$ & & Calc. $^{\mathrm{a}}$ & Expt. $^{\mathrm{b}}$ \\
\hline $\mathrm{A}_{1 \mathrm{~g}}$ & 0 & 0 & & 0 & 0 & & 0 & 0 \\
$\mathrm{~T}_{1 \mathrm{~g}}$ & 55.3 & 50 & & 29.2 & 30 & & 24.1 & 25 \\
$\mathrm{E}_{\mathrm{g}}$ & 90.0 & 88 & & 48.9 & 52 & & 40.3 & 43 \\
$\mathrm{~T}_{2 \mathrm{~g}}$ & 164.0 & 154 & & 84.5 & 87 & & 71.9 & 55 \\
\hline \hline
\end{tabular}

${ }^{\text {a The calculated parameters } F^{k}(4 f, 4 f), \zeta_{4 f} \text { and } B_{q}^{k}(4 f, 4 f) \text { of } \mathrm{Pr}^{3+} \text { within configuration }}$ $4 f^{2}$ are listed in Table $\mathrm{S} 2$ of the supplementary material.

${ }^{\mathrm{b}}$ The reference data are taken from Refs. 49, 47, and 48, which relate experimental studies of $\mathrm{Cs}_{2} \mathrm{KPrF}_{6}, \mathrm{Cs}_{2} \mathrm{NaPrCl}_{6}$, and $\mathrm{Cs}_{2} \mathrm{NaPrBr}_{6}$, respectively. 
TABLE V. Calculated Parameters (in eV) of the $\mathrm{Pr}^{3+}$ ion within configuration $3 d^{9} 4 f^{3}$ in the $\left(\mathrm{PrF}_{6}\right)^{3-},\left(\mathrm{PrCl}_{6}\right)^{3-}$, and $\left(\mathrm{PrBr}_{6}\right)^{3-}$ clusters obtained from the DFT calculation using the GGA functional. $\beta$ (in [.]) represents the ratio between the calculated parameters in the molecular clusters and free ions.

\begin{tabular}{|c|c|c|c|c|c|c|}
\hline \multirow[b]{3}{*}{$\Delta \mathrm{E}_{a v}$} & \multicolumn{2}{|c|}{$\left(\mathrm{PrF}_{6}\right)^{3-}$} & \multicolumn{2}{|c|}{$\left(\mathrm{PrCl}_{6}\right)^{3-}$} & \multicolumn{2}{|c|}{$\left(\mathrm{PrBr}_{6}\right)^{3-}$} \\
\hline & \multicolumn{2}{|c|}{$\beta$} & \multicolumn{2}{|c|}{$\beta$} & \multicolumn{2}{|c|}{$\beta$} \\
\hline & 931.47 & 0.999 & 931.29 & 0.998 & 931.18 & 0.998 \\
\hline$F^{2}(4 f, 4 f)$ & 10.882 & 0.928 & 10.160 & 0.867 & 9.851 & 0.840 \\
\hline$F^{4}(4 f, 4 f)$ & 6.790 & 0.925 & 6.335 & 0.863 & 6.140 & 0.837 \\
\hline$F^{6}(4 f, 4 f)$ & 4.874 & 0.924 & 4.546 & 0.862 & 4.405 & 0.835 \\
\hline$G^{1}(3 d, 4 f)$ & 4.514 & 0.950 & 4.334 & 0.912 & 4.253 & 0.895 \\
\hline$G^{3}(3 d, 4 f)$ & 2.672 & 0.949 & 2.565 & 0.911 & 2.517 & 0.894 \\
\hline$G^{5}(3 d, 4 f)$ & 1.853 & 0.949 & 1.780 & 0.912 & 1.746 & 0.895 \\
\hline$F^{2}(3 d, 4 f)$ & 6.918 & 0.952 & 6.653 & 0.916 & 6.534 & 0.899 \\
\hline$F^{4}(3 d, 4 f)$ & 3.111 & 0.950 & 2.989 & 0.913 & 2.933 & 0.896 \\
\hline$\zeta_{3 d}$ & 8.219 & 0.999 & 8.220 & 0.999 & 8.220 & 0.999 \\
\hline$\xi_{4 f}$ & 0.113 & 0.950 & 0.109 & 0.916 & 0.107 & 0.899 \\
\hline$B_{0}^{4}(3 d, 3 d)$ & 0.043 & $\ldots$ & 0.048 & .. & 0.050 & $\ldots$ \\
\hline$B_{0}^{4}(4 f, 4 f)$ & 0.622 & $\ldots$ & 0.388 & .. & 0.347 & $\ldots$ \\
\hline$B_{0}^{6}(4 f, 4 f)$ & 0.063 & $\ldots$ & 0.035 & $\ldots$ & 0.026 & $\ldots$ \\
\hline
\end{tabular}

functions (see also Fig. 2). Namely, the density around the $\mathrm{Pr}^{3+}$ ion, irrespective of the ligand systems, decreases over that found in the isolated atom due to migration (or expansion) of charge density from positively charged lanthanide to negatively charged ligand. In the viewpoint of ligand-field analysis, $\beta$ represents the nephelauxetic reduction of Jørgensen, ${ }^{54}$ a physical concept that enables the estimation of the strength of the Pr-ligand bonding interaction. It is observed that the values of $\beta$ are not constant (see Table V), although it is possible to define trends between equivalent parameters. That is, overall, $\beta\left(F^{k}(4 f, 4 f)\right), \beta\left(F^{k}(3 d, 4 f)\right.$ and $\left.G^{k}(3 d, 4 f)\right)$, and $\beta\left(\zeta_{4 f}^{Z O R A}\right)$ are more-or-less comparable in magnitude (see Table V). Therefore, it is derived that the nephelauxetic reduction increases in the series $\left(\mathrm{PrF}_{6}\right)^{3-}<\left(\operatorname{PrCl}_{6}\right)^{3-}<\left(\operatorname{PrBr}_{6}\right)^{3-}$ as obtained in the present work.

Figure 6 shows the calculated intensity versus excitation energies of the intra-atomic $4 f^{2}\left[\mathrm{~A}_{1 g}\right] \rightarrow 3 d^{9} 4 f^{3}$ electron transitions for $\mathrm{Pr}^{3+}$ in the three different elpasolite structures, i.e., $\mathrm{Cs}_{2} \mathrm{NaPrX}_{6}$, with $\mathrm{X}=\mathrm{F}, \mathrm{Cl}$, and $\mathrm{Br}$. The ligand-field splitting of the atomic multiplets are also taken into account using the parameters listed in Table V. For comparison, the calculated spectrum of the free $\mathrm{Pr}^{3+}$ ion is shown again [see Fig. 6(a)] with a different graphical representation than that given in Fig. 4(c). The calculated spectra in Figs. 6(b), 6(c), and 6(d) show clearly the weak influence of the ligand-field splitting of the $4 f$ orbitals, which is manifested by the acquisition of spectral profiles with substantial atomic features. Therefore it is difficult to see the variation of the absorption spectrum in terms of the strength of the ligand-field interaction, which decreases in the series $\left(\mathrm{PrF}_{6}\right)^{3-}<\left(\mathrm{PrCl}_{6}\right)^{3-}<\left(\mathrm{PrBr}_{6}\right)^{3-}$ as indicated by the listed parameters in Table V. According to Fig. 6(a), the free $\mathrm{Pr}^{3+}$ ion spectrum is composed by two absorption bands by the spin-orbit coupling interaction of the $3 d$ electrons. The first
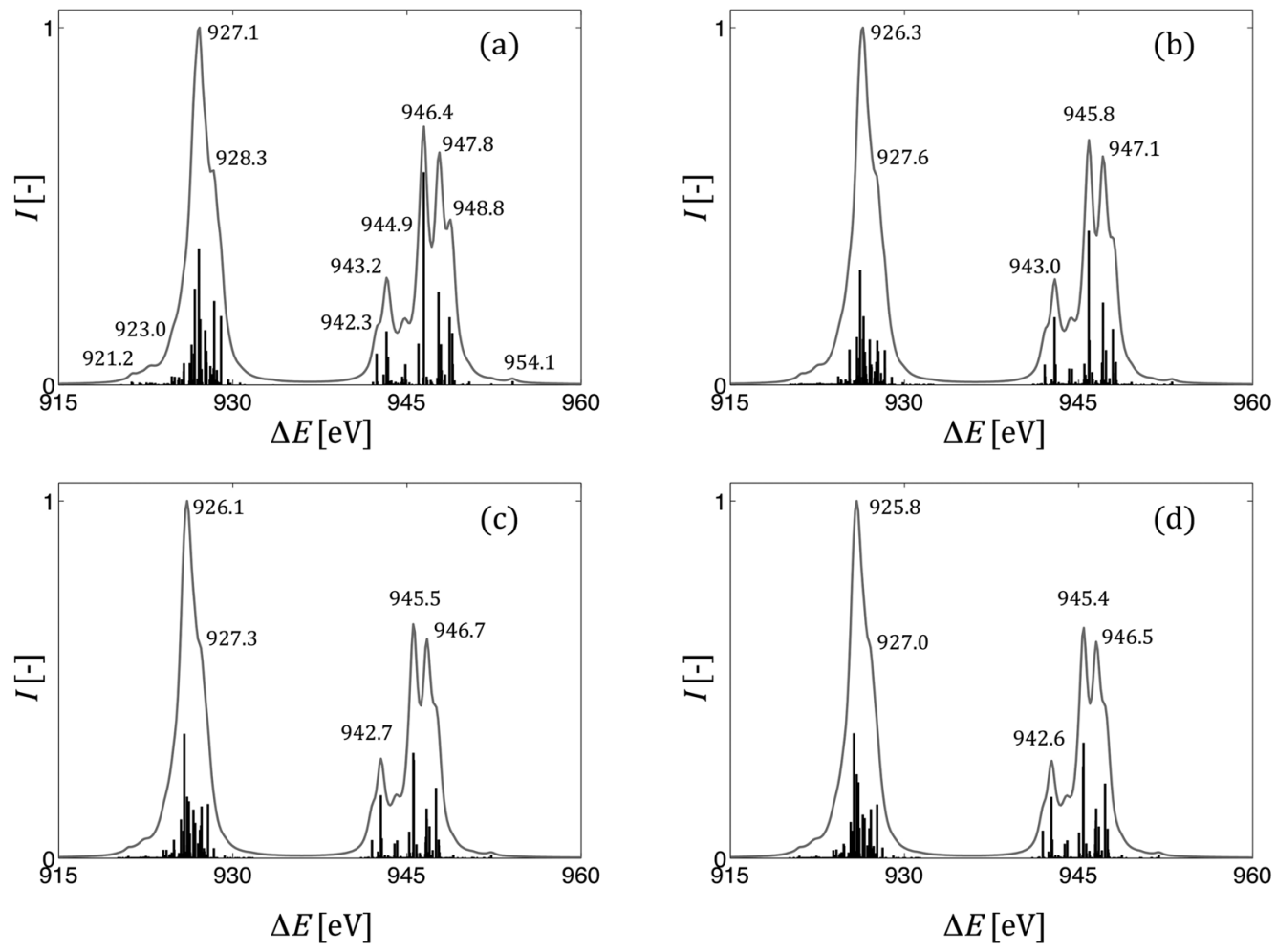

FIG. 6. Results of the modeling of the $\operatorname{Pr}^{3+} \mathrm{M}_{4,5}$-edge X-ray absorption in the free ion (a) and in the molecular $\left(\operatorname{PrF}_{6}\right)^{3-}(\mathrm{b}),\left(\operatorname{PrCl}_{6}\right)^{3-}(\mathrm{c}), \operatorname{and}^{3}\left(\operatorname{PrBr}_{6}\right)^{3-}(\mathrm{d})$ clusters, showing the calculated oscillator strengths of the intra-atomic $4 f^{2}\left[\mathrm{~A}_{1 g}\right] \rightarrow 3 d^{9} 4 f^{3}$ electron transitions within the electric-dipole approximation (black bars) and the simulated absorption spectrum (gray curves), which results from broadening of the oscillator strengths with a Lorentzian function with a constant half-width at half maximum $\left(\gamma_{i}=\gamma=0.4 \mathrm{eV}\right)$ for the all manifolds of the oscillator strengths of the $3 d^{9} 4 f^{3}$ multiplets. The intensity plot (ordinate) is given in arbitrary units. 
band, that has its maximum absorption at energy of $927.1 \mathrm{eV}$, is characterized by one strong peak and three shoulders at energies $921.2 \mathrm{eV}, 923.0 \mathrm{eV}$, and $928.3 \mathrm{eV}$ [Fig. 6(a)]. The second band has more complex features; it splits into three peaks at energies $943.2 \mathrm{eV}, 946.4 \mathrm{eV}$, and $947.8 \mathrm{eV}$ with four shoulders at energies $942.3 \mathrm{eV}, 944.9 \mathrm{eV}, 948.8 \mathrm{eV}$, and $954.1 \mathrm{eV}$ [Fig. 6(a)]. By taking into account the ligand-field splitting, the spherical symmetry of the atomic system is reduced, giving rise to the mixing between final states in the $3 d^{9} 4 f^{3}$ multiplets. Although the energy splitting of the atomic multiplet terms of $\mathrm{Pr}^{3+}$ is rather small for the three systems under consideration, it is observed that small features, which exist in the free ion spectra, tend to disappear in the $\left(\mathrm{PrF}_{6}\right)^{3-},\left(\mathrm{PrCl}_{6}\right)^{3-}$, and $\left(\operatorname{PrBr}_{6}\right)^{3-}$ spectra. For example, the main peaks that are observed in the spectra are shifted to lower energy, and some of the shoulders that are formally obtained in the free ion are no longer observed in Figs. 6(b), 6(c), and 6(d).

\section{CONCLUSIONS}

The theoretical model that is reported in the present work is a non-empirical tool that enables the calculation of lanthanide $\mathrm{M}_{4,5}$-edge $\mathrm{X}$-ray absorption spectra. An effective ligand-field Hamiltonian is operated in conjunction with first principles Density-Functional Theory (DFT) calculation in order to obtain non-empirically the excitation energies and oscillator strengths of the intra-atomic $4 f^{n} \rightarrow 3 d^{9} 4 f^{n+1}$ electron transitions within the electric-dipole approximation. The DFT-based theoretical model allows the estimation of the Slater-Condon integrals, spin-orbit coupling constants, and ligand-field potential that parameterize the inter-electronic repulsion, relativistic spin-orbit interaction, and chemical ligand-coordination of the lanthanide ions. The test of the influence of the DFT functional, including LDA, GGA, and hybrid, reveals a priori minor variation in the parameter calculations corresponding to the isolated lanthanide ions (see Table II).

The present work is aimed at providing a validation of the theoretical concept by proposing examples for the application and performing comparative analysis with available experimental data. In the free ion systems, the Slater-Condon parameters are overestimated by the DFT calculation. The percentage error is relatively important by typically $10 \%-20 \%$ over the reference values (see Fig. 3). However, the calculated X-ray absorption spectra are relatively good despite the slight energy shift in the calculated excitation energies (see Fig. 4). In the solid-state lanthanide systems, practical limitation of the theoretical model renders the evaluation of bulk crystal structures yet impossible. Therefore, molecular clusters are constructed that approximately represent the local environment of the $\mathrm{Pr}^{3+}$ ion in the elpasolite $\mathrm{Cs}_{2} \mathrm{NaPrF}_{6}, \mathrm{Cs}_{2} \mathrm{NaPrCl}_{6}$, and $\mathrm{Cs}_{2} \mathrm{NaPrBr}_{6}$ structures. The calculated Slater-Condon integrals are reduced by about $95 \%\left(\mathrm{Cs}_{2} \mathrm{NaPrF}_{6}\right), 91 \%\left(\mathrm{Cs}_{2} \mathrm{NaPrCl}_{6}\right)$, and $89 \%$ $\left(\mathrm{Cs}_{2} \mathrm{NaPrBr}_{6}\right)$ over the atomic parameters (see Table V). Hence the screening of the inter-electron correlation is quantitatively evaluated, enabling also the calculation procedure without empirical corrections or scaling factors. The calculated X-ray absorption spectra of $\mathrm{Cs}_{2} \mathrm{NaPrF}_{6}, \mathrm{Cs}_{2} \mathrm{NaPrCl}_{6}$, and $\mathrm{Cs}_{2} \mathrm{NaPrBr}_{6}$ maintain substantial atomic features (see
Fig. 6). However, the consideration of the ligand-field splitting of the atomic multiplet energies leads to the observation of tiny details: e.g., some shoulders in the atomic spectra tend to disappear. This observation is inferred from the redistribution of the intensity onto the ligand-field manifold of the atomic multiplet levels of configuration $3 d^{9} 4 f^{3}$.

The present theoretical model ("LFDFT for X-ray spectroscopies" was instigated in the Paul Scherrer Institute during the time period 2015-2018) responds to the practical need given the availability of the large-scale Synchrotron facility "Swiss Light Source." By making this presentation, my intention is to show that the spectral profiles of lanthanide $\mathrm{M}_{4,5}$-edge X-ray absorption can be determined form first principles DFT calculation and to advertise the recently available LFDFT tool in the Amsterdam Density Functional (ADF) program package. Future studies include new methodological development in relation to the magnetic dichroism in X-ray spectroscopies and selective application in relation to the characterization of technological materials. That is, a strong theoretical experience is ultimately a good complement to experimental studies, paving the way for a better understanding of the optical properties of materials and for a good knowledge of the mechanism of core-electron excitation.

\section{SUPPLEMENTARY MATERIAL}

See supplementary material for the graphical representations of the radial functions of the $3 d$ and $4 f$ KohnSham orbitals of trivalent lanthanide ions within configuration $3 d^{9} 4 f^{n+1}$; the tabulated values of the parameters $F^{k}(4 f, 4 f)$ and $\zeta_{4 f}$ of trivalent lanthanide ions within the ground configuration $4 f^{n}$ obtained from DFT; the high quality color figures for the representation of the excitation energies and oscillator strengths of the intra-atomic $4 f^{n} \rightarrow 3 d^{9} 4 f^{n+1}$ electron transitions within the electric-dipole approximation; the tabulated values of the parameters $F^{k}(4 f, 4 f), \zeta_{4 f}$ and $B_{q}^{k}(4 f, 4 f)$ of the $\mathrm{Pr}^{3+}$ ion within the ground configuration $4 f^{2}$ in the elpasolite $\mathrm{Cs}_{2} \mathrm{NaPrX}_{6}$, with $\mathrm{X}=\mathrm{F}, \mathrm{Cl}$, and $\mathrm{Br}$; and the energy diagram for Kohn-Sham orbitals of the studied molecular $\left(\operatorname{PrX}_{6}\right)^{3-}$ cluster, with $\mathrm{X}=\mathrm{F}, \mathrm{Cl}$, and $\mathrm{Br}$.

\section{ACKNOWLEDGMENTS}

It is my pleasure to recognize useful discussions with Professor Dr. Claude Daul (University of Fribourg in Switzerland). I am thankful to Dr. Jan Dreiser (Paul Scherrer Institute in Switzerland) for his interest in the theoretical project. I am also thankful to Dr. Goutam Kuri and Dr. Johannes Bertsch (Paul Scherrer Institute in Switzerland) for their trust and constant support. I am indebted to SCM (www.scm.com) for the invitation to the ADF developer meeting 2018 in Amsterdam. This work is partly supported by Swissnuclear.

\footnotetext{
${ }^{1}$ J.-C. Bünzli and C. Piguet, Chem. Soc. Rev. 34, 1048 (2005).

${ }^{2}$ H. Winick, J. Syncrotron Radiat. 5, 168 (1998).

${ }^{3}$ D. H. Bilderback, P. Elleaume, and E. Weckert, J. Phys. B: At., Mol. Opt. Phys. 38, S773 (2005).

${ }^{4}$ F. de Groot, Chem. Rev. 101, 1779 (2001).

${ }^{5}$ F. de Groot, Coord. Chem. Rev. 249, 31 (2005).

${ }^{6}$ M. E. Casida and M. Huix-Rotlant, Annu. Rev. Phys. Chem. 63, 287 (2012).
} 
${ }^{7}$ M. Stener, G. Fronzoni, and M. de Simone, Chem. Phys. Lett. 373, 115 (2003).

${ }^{8}$ B. T. Thole, G. van der Laan, J. C. Fuggle, G. A. Sawatzky, R. C. Karnatak, and J. M. Esteva, Phys. Rev. B 32, 5107 (1985).

${ }^{9}$ H. Bethe, Ann. Phys. 395, 133 (1929).

${ }^{10}$ J. H. van Vleck, J. Chem. Phys. 3, 807 (1935).

${ }^{11}$ G. te Velde, F. M. Bickelhaupt, E. J. Baerends, C. Fonceca-Guerra, S. J. A. van Gisbergen, J. G. Snijders, and T. Ziegler, J. Comput. Chem. 22, 931 (2001).

${ }^{12}$ C. Fonseca-Guerra, J. G. Snijders, G. te Velde, and E. J. Baerends, Theor. Chem. Acc. 99, 391 (1998).

${ }^{13}$ E. J. Baerends, T. Ziegler, A. J. Atkins, J. Autschbach, O. Baseggio, D. Bashford, A. Berces, F. M. Bickelhaupt, C. Bo, P. M. Boerrigter, L. Cavallo, C. Daul, D. P. Chong, D. V. Chulhai, L. Deng, R. M. Dickson, J. M. Dieterich, D. E. Ellis, M. van Faassen, L. Fan, T. H. Fischer, C. Fonseca Guerra, M. Franchini, A. Ghysels, A. Giammona, S. J. A. van Gisbergen, A. Goez, A. W. Götz, J. A. Groeneveld, O. V. Gritsenko, M. Grüning, S. Gusarov, F. E. Harris, P. van den Hoek, Z. Hu, C. R. Jacob, H. Jacobsen, L. Jensen, L. Joubert, J. W. Kaminski, G. van Kessel, C. König, F. Kootstra, A. Kovalenko, M. V. Krykunov, E. van Lenthe, D. A. McCormack, A. Michalak, M. Mitoraj, S. M. Morton, J. Neugebauer, V. P. Nicu, L. Noodleman, V. P. Osinga, S. Patchkovskii, M. Pavanello, C. A. Peeples, P. H. T. Philipsen, D. Post, C. C. Pye, H. Ramanantoanina, P. Ramos, W. Ravenek, J. I. Rodriguez, P. Ros, R. Rüger, P. R. T. Schipper, D. Schlüns, H. van Schoot, G. Schreckenbach, J. S. Seldenthuis, M. Seth, J. G. Snijders, M. Sola, M. Stener, M. Swart, D. Swerhone, V. Tognetti, G. te Velde, P. Vernooijs, L. Versluis, L. Visscher, O. Visser, F. Wang, T. A. Wesolowski, E. M. van Wezenbeek, G. Wiesenekker, S. K. Wolff, T. K. Woo and A. L. Yakovlev, ADF2017, available at http://www.scm.com, 2017.

${ }^{14}$ M. Atanasov, C. A. Daul, and C. Rauzy, Struct. Bonding 106, 97 (2004).

${ }^{15} \mathrm{M}$. Atanasov, C. Rauzy, P. Baettig, and C. Daul, Int. J. Quantum Chem. 102, 119 (2005).

${ }^{16}$ H. Ramanantoanina, W. Urland, F. Cimpoesu, and C. Daul, Phys. Chem. Chem. Phys. 15, 13902 (2013).

${ }^{17}$ H. Ramanantoanina, M. Sahnoun, A. Barbiero, M. Ferbinteanu, and F. Cimpoesu, Phys. Chem. Chem. Phys. 17, 18547 (2015).

${ }^{18}$ H. Ramanantoanina, W. Urland, B. Herden, F. Cimpoesu, and C. Daul, Phys. Chem. Chem. Phys. 17, 9116 (2015).

${ }^{19}$ H. Ramanantoanina, F. Cimpoesu, C. Göttel, M. Sahnoun, B. Herden, M. Suta, C. Wickleder, W. Urland, and C. Daul, Inorg. Chem. 54, 8319 (2015).

${ }^{20}$ F. Cimpoesu, B. Frecus, C. I. Oprea, H. Ramanantoanina, W. Urland, and C. Daul, Mol. Phys. 113, 1712 (2015).

${ }^{21}$ T. Ziegler, A. Rauk, and E. J. Baerends, Theor. Chim. Acta 43, 261 (1977).

${ }^{22}$ J. P. Perdew, K. Burke, and M. Ernzerhof, Phys. Rev. Lett. 77, 3865 (1996).

${ }^{23}$ S. H. Vosko, L. Wilk, and M. Nusair, Can. J. Phys. 58, 1200 (1980).

${ }^{24}$ P. J. Stephens, F. J. Devlin, C. F. Chabalowski, and M. J. Frisch, J. Phys. Chem. 98, 11623 (1994)

${ }^{25}$ E. van Lenthe and E. J. Baerends, J. Comput. Chem. 24, 1142 (2003).
${ }^{26}$ C. Chang, M. Pelissier, and P. Durant, Phys. Scr. 34, 394 (1986).

${ }^{27}$ E. van Lenthe, E. J. Baerends, and J. G. Snijders, J. Chem. Phys. 99, 4597 (1993).

${ }^{28}$ C. Daul, Int. J. Quantum Chem. 52, 867 (1994).

${ }^{29}$ R. D. Cowan, The Theory of Atomic Structure and Spectra (University of California Press, Berkeley, 1981).

${ }^{30}$ B. R. Judd, Operator Techniques in Atomic Spectroscopy (Princeton University Press, Princeton, 1998).

31 J. C. Slater, Phys. Rev. 34, 1293 (1929).

${ }^{32}$ H. Ramanantoanina, W. Urland, F. Cimpoesu, and C. Daul, Phys. Chem. Chem. Phys. 16, 12282 (2014).

${ }^{33}$ B. G. Wybourne, Phys. Rev. 148, 317 (1966).

${ }^{34}$ J. S. Griffith, The Theory of Transition Metal Ions (Cambridge University Press, Cambridge, 1961).

${ }^{35}$ C. J. Balhausen, Introduction to Ligand Field Theory (McGraw-Hill Book Co., New York, 1962).

${ }^{36}$ H. Ramanantoanina and C. Daul, J. Mol. Model. 23, 243 (2017).

${ }^{37}$ H. Ramanantoanina and C. Daul, Phys. Chem. Chem. Phys. 19, 20919 (2017).

${ }^{38}$ H. Ramanantoanina, G. Kuri, C. Daul, and J. Bertsch, Phys. Chem. Chem. Phys. 18, 19020 (2016).

${ }^{39}$ H. Ramanantoanina, Phys. Chem. Chem. Phys. 19, 32481 (2017).

${ }^{40}$ E. van Lenthe, E. J. Baerends, and J. G. Snijders, J. Chem. Phys. 101, 9783 (1994).

${ }^{41}$ D. J. Newman and B. K. C. Ng, Crystal Field Handbook (Cambridge University Press, Cambridge, 2000).

${ }^{42}$ M. O. Krause and J. H. Olivier, J. Phys. Chem. Ref. Data 8, 329 (1979).

${ }^{43}$ W. C. Martin, R. Zalubas and L. Hagan, Natl. Stand. Ref. Data Ser., Natl. Bur. Stand. (U.S.) Monogr. 60 (U.S. Government Printing Office, 1978), $422 \mathrm{p}$.

${ }^{44}$ A. Kramida, Y. Ralchenko, J. Reader, and NIST ASD Team, http://physics.nist-gov/asd (accessed May 28, 2018).

${ }^{45}$ F. M. F. de Groot, J. C. Fuggle, B. T. Thole, and G. A. Sawatzky, Phys. Rev. B 42, 5459 (1990).

${ }^{46}$ C.-K. Duan, P. A. Tanner, V. Makhov, and N. Khaidukov, J. Phys. Chem. A 115, 8870 (2011).

${ }^{47}$ F. S. Richardson, M. F. Reid, J. J. Dallara, and R. D. Smith, J. Chem. Phys. 83, 3813 (1985).

${ }^{48}$ A. Furrer and H.-U. Güdel, Phys. Rev. B 56, 15062 (1997).

${ }^{49}$ P. A. Tanner, V. V. Ravi Kanth Kumar, C. K. Jayasankar, and M. F. Reid, J. Alloys Compd. 215, 349 (1994).

${ }^{50} \mathrm{R}$. Acevedo and V. Poblete, Powder Diffr. 10, 241 (1995).

${ }^{51}$ D. Aravena, M. Atanasov, and F. Neese, Inorg. Chem. 55, 4457 (2016).

${ }^{52}$ M. Atanasov, C. Daul, H. U. Güdel, T. A. Weselowski, and M. Zbiri, Inorg. Chem. 44, 2954 (2005).

${ }^{53}$ R. D. Shannon, Acta Crystallogr., Sect. A: Cryst. Phys. Diffr., Theor. Gen. Crystallogr. 32, 751 (1976).

${ }^{54}$ C. K. Jørgensen, Modern Aspects of Ligand-Field Theory (North-Holland Publishing Co., 1971). 